\title{
Universal anisotropic finite-size critical behavior of the two-dimensional Ising model on a strip and of $d$-dimensional models on films
}

\author{
Boris Kastening* \\ Institute for Materials Science, Technische Universität Darmstadt, 64287 Darmstadt, Germany
}

(Dated: August 16, 2021)

\begin{abstract}
Anisotropy effects on the finite-size critical behavior of a two-dimensional Ising model on a general triangular lattice in an infinite-strip geometry with periodic, antiperiodic, and free boundary conditions (bc) in the finite direction are investigated. Exact results are obtained for the scaling functions of the finite-size contributions to the free energy density. With $\xi_{>}$the largest and $\xi_{<}$the smallest bulk correlation length at a given temperature near criticality, we find that the dependence of these functions on the ratio $\xi_{<} / \xi_{>}$and on the angle parameterizing the orientation of the correlation volume is of geometric nature. Since the scaling functions are independent of the particular microscopic realization of the anisotropy within the two-dimensional Ising model, our results provide a limited verification of universality. We explain our observations by considering finite-size scaling of free energy densities of general weakly anisotropic models on a $d$-dimensional film, i.e., in an $L \times \infty^{d-1}$ geometry, with bc in the finite direction that are invariant under a shear transformation relating the anisotropic and isotropic cases. This allows us to relate free energy scaling functions in the presence of an anisotropy to those of the corresponding isotropic system. We interpret our results as a simple and transparent case of anisotropic universality, where, compared to the isotropic case, scaling functions depend additionally on the shape and orientation of the correlation volume. We conjecture that this universality extends to cases where the geometry and/or the bc are not invariant under the shear transformation and argue in favor of validity of two-scale factor universality for weakly anisotropic systems.
\end{abstract}

PACS numbers: 64.60.an, 05.50.+q, 05.70.Jk, 64.60.De, 64.60.F-, 68.35.Rh

Keywords: Ising model; anisotropy; universality; free energy; film geometry; critical point; finite-size scaling; scaling function, Casimir amplitude

\section{INTRODUCTION}

Bulk critical phenomena can be divided into distinct universality classes [1]. Critical exponents, certain critical amplitude ratios, and the near-critical behavior of thermodynamic functions are identical for the members of such a universality class and are called universal, since they depend only on macroscopic properties such as near-critical correlation lengths and not on the microscopic details of the system under consideration. For instance, the correlation length in the asymptotic critical domain, i.e., for asymptotically small positive or negative $t \equiv\left(T-T_{\mathrm{c}}\right) / T_{\mathrm{c}}$, is, for isotropic systems, described by

$$
\xi=\xi_{ \pm, 0}|t|^{-\nu}, \quad T \gtrless T_{\mathrm{c}},
$$

where $T_{\mathrm{c}}$ is the bulk critical temperature, $\nu$ is a universal critical exponent, $\xi_{ \pm, 0}$ are nonuniversal critical amplitudes, and $R_{\xi} \equiv \xi_{+, 0} / \xi_{-, 0}$ is a universal critical amplitude ratio.

An extension to the concept of bulk universality concerns systems that are geometrically confined on a length scale $L$ in one or more directions, where $L$ is large compared to all microscopic length scales of the system, such as lattice spacings (see [2-4]; for reviews see, e.g., $[5,6])$. Consider the free energy density $f$ in units of

*Email address: bkastening@matgeo.tu-darmstadt.de
$k_{\mathrm{B}} T$ (this normalization is used without further mentioning for all free energy densities throughout this work) of a $d$-dimensional system that is isotropic at such large distances. Assume that $f$ may be split uniquely into a nonsingular and a singular contribution according to

$$
f(T, L)=f_{\mathrm{ns}}(T, L)+f_{\mathrm{s}}(t, L),
$$

where the singular contribution $f_{\mathrm{s}}$ is defined as that part of $f$ that becomes singular in $t$ at $t=0$ in the bulk limit $L \rightarrow \infty$. If $f_{s}$ exhibits scaling, its behavior in the asymptotic critical domain of large $L$ and small $|t|$, where Wegner corrections to scaling [7] are negligible, may be described by a scaling function $\mathcal{F}$ according to

$$
L^{d} f_{s}(t, L)=\mathcal{F}(\tilde{x}),
$$

with the scaling variable $\tilde{x} \equiv\left(L / \xi_{+, 0}\right)^{1 / \nu} t$. If $\mathcal{F}$ exists, it is expected to be universal. It describes the scaling behavior of the asymptotic singular part of the free energy density for given system geometry and boundary conditions (bc) for the bulk universality class under consideration for the isotropic case.

With the bulk free energy density

$$
f_{\mathrm{b}}(T) \equiv \lim _{L \rightarrow \infty} f(T, L),
$$

we may split the free energy density according to

$$
\begin{aligned}
f(T, L) & =f_{\mathrm{b}}(T)+f_{\mathrm{ex}}(T, L), \\
f_{\mathrm{ex}}(T, L) & =f_{\mathrm{sf}}(T, L)+f_{\mathrm{fs}}(T, L),
\end{aligned}
$$


where $f_{\text {ex }}$ is called the excess free energy density, $f_{\mathrm{sf}}(T, L)=L^{-1} \bar{f}_{\mathrm{sf}}(T)$ represents any surface or interface contributions, and $f_{\mathrm{fs}}$ is called the finite-size contribution to the free energy density. Denote the singular parts of $f_{\mathrm{ex}}, f_{\mathrm{sf}}$, and $f_{\mathrm{fs}}$ by $f_{\mathrm{ex}, \mathrm{s}}, f_{\mathrm{sf}, \mathrm{s}}$, and $f_{\mathrm{fs}, \mathrm{s}}$, respectively. If the leading contributions to these singular parts exhibit scaling, we may write in the asymptotic critical domain

$$
\begin{aligned}
L^{d} f_{\mathrm{ex}, \mathrm{s}}(t, L) & =\mathcal{A}(\tilde{x}), \\
L^{d} f_{\mathrm{fs}, \mathrm{s}}(t, L) & =\mathcal{G}(\tilde{x}),
\end{aligned}
$$

with scaling functions $\mathcal{A}$ and $\mathcal{G}$. These, again, are expected to be universal. The Casimir amplitude is defined as the critical value of $\mathcal{G}$, i.e., by

$$
\Delta \equiv \mathcal{G}(0) .
$$

The physical motivation for investigating the scaling functions $\mathcal{A}$ and $\mathcal{G}$ and the Casimir amplitude lies in their close connection to the critical Casimir force and its scaling function, see, e.g., the monographs [8, 9], and the recent overview article [10] (see also Appendix B). Ref. [10] also provides a collection of recent theoretical and experimental results.

Now consider weakly anisotropic systems, which are characterized by a single bulk correlation-length exponent $\nu$. We assume here that their bulk correlation lengths are related by a shear transformation to the isotropic case. Then $\nu$ assumes in any direction the same value as for the isotropic case, which is supported by other investigations [11-15]. On the other hand, the $\xi_{ \pm, 0}$ depend on the direction and are, for a $d$-dimensional system, described by the surface of a $d$-dimensional ellipsoid. However, in any given direction, Eq. (1) holds and $R_{\xi}$ assumes its isotropic value.

The situation is less clear for the scaling functions on the right hand sides of Eqs. (3) and (6), since they may additionally depend on the parameters describing the shape and the orientation of the correlation lengths ellipsoid, i.e., on $d-1$ correlation length ratios and $d(d-1) / 2$ angles. These additional $d(d+1) / 2-1$ parameters may be organized into a $d \times d$ symmetric matrix $\overline{\mathbf{A}}$ with $\operatorname{det} \overline{\mathbf{A}}=1$ [16]. The question arises whether such a function can be considered universal [17-19]. Dohm [18] interprets any dependence on $\overline{\mathbf{A}}$ as nonuniversal. Diehl and Chamati [19] suggest to define universality only after transforming to an isotropic system by means of a shear transformation. Here we advocate the interpretation that scaling functions even for weakly anisotropic systems are universal, if they depend on the anisotropy only through its long-distance properties parameterized by $\overline{\mathbf{A}}$ and not on any microscopic details of how it is realized. We will return to this issue in Sec. IV.

For simplicity, we consider only systems, where the transformation to an isotropic system leaves the geometry and the bc invariant. In particular, we compute free energy scaling functions for the specific case of an anisotropic two-dimensional Ising model on an infinite strip of width $L$ and explain our explicit exact results by investigating systems confined to $d$-dimensional films of width $L$. We consider periodic, antiperiodic, fixed, and free bc in the direction of the width $L$ of the film. For all cases we define a scaling variable by

$$
\tilde{x} \equiv\left(L / \xi_{+, 0}^{(L)}\right)^{1 / \nu} t,
$$

where $\xi_{+, 0}^{(L)}$ is the $T>T_{\mathrm{c}}$ amplitude of the bulk correlation length $\xi_{L}$ perpendicular to the film boundaries with a corresponding asymptotic critical behavior

$$
\xi_{L}=\xi_{ \pm, 0}^{(L)}|t|^{-\nu}, \quad T \gtrless T_{\mathrm{c}},
$$

where $\xi_{+, 0}^{(L)} / \xi_{-, 0}^{(L)}=R_{\xi}$.

We do not consider complications arising from strong anisotropies [20], from subleading long-range interactions $[19,21]$, or from scaling violations for large $\tilde{x}$ arising in a region of large $L$ for fixed $\xi_{L}$ that manifest themselves in a nonuniform convergence of the leading singular part of free energy densities towards the respective scaling function in the asymptotic critical domain [22].

This work is structured as follows. In Sec. II, we discuss the two-dimensional Ising model on a triangular lattice in infinite-strip geometry. In Sec. II A, the model is set up and basic quantities are defined. In Sec. IIB, we recall explicit results for the bulk correlation lengths and discuss their behavior near $T_{\mathrm{c}}$. In Sec. IIC, we derive the scaling behavior of the singular contributions to $f_{\mathrm{ex}}$, $f_{\mathrm{sf}}$, and $f_{\mathrm{fs}}$ for periodic, antiperiodic (Sec. II C 1 ), and free (Sec. II C 2) bc to the extent they are defined. We derive explicit expressions for the corresponding scaling functions and critical Casimir amplitudes. In Sec. III, we discuss the finite-size scaling behavior for free energy densities of $d$-dimensional anisotropic films with periodic and antiperiodic bc (Sec. III B 1) and fixed and free bc (Sec. III B 2) and compare our results to those found in Sec. II C. The discussion of the results of Secs. II and III is delegated to Sec. IV.

\section{TWO-DIMENSIONAL ISING MODEL}

\section{A. Basic definitions}

Consider a two-dimensional Ising model on a general triangular lattice with lattice constants $d_{i}$ along the three lattice directions, see Fig. 1. For simplicity, we assume a ferromagnetic model with only nearest-neighbor couplings $J_{i}$ along the sides of length $d_{i}$. Consider this model on an $L_{1} \times L_{23}$ rectangular geometry, with the $L_{1}$ direction parallel to the " 1 " lattice direction. Let there be $N_{1}$ layers in the " 1 " direction and $N_{23}$ layers in the " 23 " direction perpendicular to the "1" direction, so that

$$
\begin{aligned}
L_{1} & =N_{1} d_{1}, \\
L_{23} & =N_{23} h_{1},
\end{aligned}
$$

where $h_{1}$ is the height of the elementary triangle with respect to the side of length $d_{1}$, see Fig. 1. 


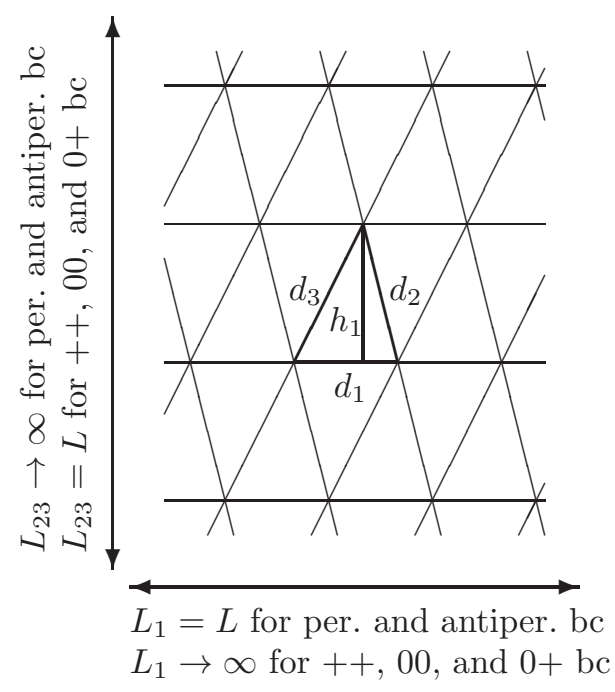

FIG. 1: Triangular lattice with lattice constants $d_{i}$ on an infinitely long strip of width $L$.

Periodic or antiperiodic bc are easiest implemented along one of the three lattice directions. We want this direction to be parallel to one of the rectangle edges and therefore choose the "1" direction. Periodic bc are imposed by identifying a line of spins along the "2" direction (or, equivalently, the "3" direction) with spins along such a line at a distance $L_{1}$ in the "1" direction (one may think of the lattice as wrapped around a cylinder of circumference $L_{1}$, whose axis points along the "23" direction). For antiperiodic bc, in addition the signs of the couplings $J_{1}$ along one side of one such line are reversed.

Both free ("0") bc, i.e., no further neighboring spin for the last spins on one edge of the rectangle, and fixed ("+") bc, i.e., a fictitious neighboring spin with fixed value +1 for each last spin on one edge of the rectangle, are easiest implemented if such an edge is along one of the lattice directions. For the rectangle defined above, this leads to the unique choice of imposing " 00 ," " ++ ," or " $0+$ " bc in the " 23 " direction, where the two entries refer to the two opposite sides of the rectangle.

With $\beta \equiv 1 /\left(k_{\mathrm{B}} T\right)$ and $K_{i} \equiv \beta J_{i}$, the Hamiltonian of this model reads

$$
\begin{gathered}
-\beta H=\sum_{m, n}\left(K_{1} s_{m, n} s_{m+1, n}+K_{2} s_{m, n} s_{m, n+1}\right. \\
\left.+K_{3} s_{m, n} s_{m+1, n+1}\right) .
\end{gathered}
$$

With the partition function

$$
Z\left(T, L_{1}, L_{23}\right)=\sum_{\left\{s_{i, j}= \pm 1\right\}} e^{-\beta H},
$$

the rectangle free energy density is given by

$$
f_{\text {rect }}\left(T, L_{1}, L_{23}\right)=-\left(L_{1} L_{23}\right)^{-1} \ln Z\left(T, L_{1}, L_{23}\right) .
$$

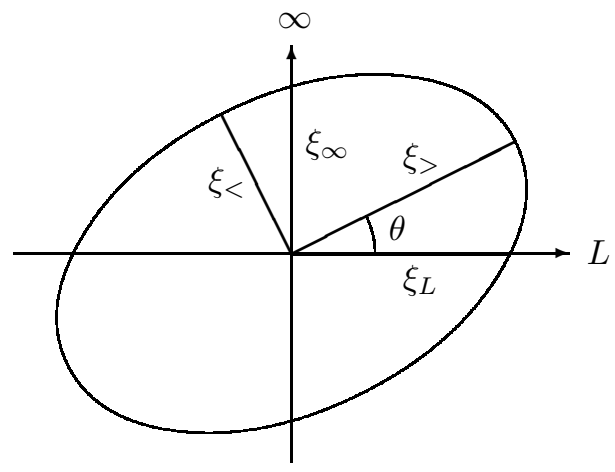

FIG. 2: Bulk correlation lengths ellipse with largest and smallest correlation lengths $\xi_{>}$and $\xi_{<}$, respectively. The $L$ direction is the direction perpendicular to the infinite direction of the strip and $\xi_{L}$ and $\xi_{\infty}$ are the bulk correlation lengths in these directions.

We are interested in the free energy density

$$
f(T, L) \equiv \lim _{L_{\|} \rightarrow \infty} \begin{cases}f_{\text {rect }}\left(T, L, L_{\|}\right) & \text {for per., antiper. bc, } \\ f_{\text {rect }}\left(T, L_{\|}, L\right) & \text { for }++, 00,0+\text { bc }\end{cases}
$$

of an infinitely long strip, i.e., for an $L \times \infty$ geometry, with periodic, antiperiodic, $00,++$, or $0+$ bc in the $L$ direction. For the width $L$ of the strip holds

$$
L= \begin{cases}L_{1} & \text { for periodic and antiperiodic bc } \\ L_{23} & \text { for }++, 00, \text { and } 0+\mathrm{bc}\end{cases}
$$

and the same relation between $N, N_{1}$, and $N_{23}$.

\section{B. Bulk correlation lengths}

For two-dimensional systems, the ellipsoid of Sec. I describing the bulk correlation lengths in the asymptotic critical domain reduces to an ellipse, having a major radius $\xi_{>}$and a minor radius $\xi_{<}$. Define a bulk correlation "volume" by

$$
V_{\text {co }} \equiv \xi_{>} \xi_{<}
$$

and an aspect ratio by

$$
r \equiv \xi_{<} / \xi_{>}, \quad 0<r \leq 1 .
$$

Let the direction of the major radius be rotated by an angle $\theta$ with respect to the $L$ direction of the infinitely long strip, see Fig. 2.

Call $\xi_{\infty}$ the bulk correlation length in the infinitelength direction of the strip and $\xi_{L}$ the bulk correlation length in the perpendicular direction. Basic geometric considerations provide the relations

$$
\begin{aligned}
& \xi_{\infty}^{-2}=\xi_{>}^{-2} \sin ^{2} \theta+\xi_{<}^{-2} \cos ^{2} \theta \\
& \xi_{L}^{-2}=\xi_{>}^{-2} \cos ^{2} \theta+\xi_{<}^{-2} \sin ^{2} \theta
\end{aligned}
$$


between the various correlation lengths in the asymptotic critical domain.

Let $\xi_{i}, i=1,2,3$ be the bulk correlation lengths in the $i$ th lattice direction. It follows that

$$
\xi_{1}= \begin{cases}\xi_{L} & \text { for periodic and antiperiodic bc, } \\ \xi_{\infty} & \text { for }++, 00, \text { and } 0+\mathrm{bc} .\end{cases}
$$

Generalizing (1), the bulk correlation lengths behave, in the asymptotic critical domain, according to

$$
\xi_{i}=\xi_{ \pm, 0}^{(i)}|t|^{-\nu} \quad T \gtrless T_{\mathrm{c}},
$$

with $\xi_{+, 0}^{(i)} / \xi_{-, 0}^{(i)}=R_{\xi}$. For the two-dimensional Ising model, well-known exact results are

$$
\nu=1, \quad R_{\xi}=2 .
$$

According to Eq. (A22) of Ref. [23], the $T>T_{\mathrm{c}}$ asymptotic bulk correlation lengths are given by [24]

$$
d_{i} / \xi_{i}=-\ln \gamma^{(i)}
$$

where

$$
\gamma^{(i)} \equiv \frac{a^{(i)}+\sqrt{a^{(i)^{2}}-4 b^{(i)} c^{(i)}}}{2 c^{(i)}},
$$

with

$$
\begin{aligned}
a^{(i)} & \equiv 2 z_{i}\left(1+z_{j}^{2}\right)\left(1+z_{k}^{2}\right)+4 z_{j} z_{k}\left(1+z_{i}^{2}\right) \\
b^{(i)} & \equiv z_{i}^{2}\left(1-z_{j}^{2}\right)\left(1-z_{k}^{2}\right) \\
c^{(i)} & \equiv\left(1-z_{j}^{2}\right)\left(1-z_{k}^{2}\right)
\end{aligned}
$$

with $i, j, k=1,2,3$ or cyclic permutations, and with

$$
z_{i} \equiv \tanh K_{i}
$$

At the critical point the bulk correlation lengths diverge and therefore $\gamma^{(i)}=1$. This condition may be written as

$$
z_{1}+z_{2}+z_{3}+z_{2} z_{3}+z_{3} z_{1}+z_{1} z_{2}-z_{1} z_{2} z_{3}=1
$$

so that

$$
z_{1, \mathrm{c}}\left(z_{2}, z_{3}\right)=\frac{1-z_{2} z_{3}-z_{2}-z_{3}}{1-z_{2} z_{3}+z_{2}+z_{3}}
$$

is the critical value of $z_{1}$ for given $z_{2}$ and $z_{3}$. Combining (20)-(23), we may expand around the critical point through linear order in $t$ resulting in

$$
z_{1}=z_{1, \mathrm{c}}\left(z_{2}, z_{3}\right)-\frac{\left(1-z_{2}^{2}\right)\left(1-z_{3}^{2}\right)}{\left(1-z_{2} z_{3}+z_{2}+z_{3}\right)^{2}} \frac{d_{1} t}{\xi_{+, 0}^{(1)}}
$$

and relate the $\xi_{i}$ according to

$$
\frac{d_{1} / \xi_{1}}{2\left(1-z_{2} z_{3}\right)\left(z_{2}+z_{3}\right)}=\frac{d_{2} / \xi_{2}}{\left(1-z_{2}^{2}\right)\left(1+z_{3}^{2}\right)}=\frac{d_{3} / \xi_{3}}{\left(1-z_{3}^{2}\right)\left(1+z_{2}^{2}\right)},
$$

where terms of higher than linear order in $t$ have been omitted.

Geometric considerations, which we do not reproduce here, yield

$$
V_{\mathrm{co}}=\frac{A_{\mathrm{tr}}\left(d_{1}, d_{2}, d_{3}\right)}{A_{\mathrm{tr}}\left(d_{1} / \xi_{1}, d_{2} / \xi_{2}, d_{3} / \xi_{3}\right)},
$$

where the function

$$
A_{\mathrm{tr}}\left(\ell_{1}, \ell_{2}, \ell_{3}\right)=\frac{1}{4} \sqrt{2\left(\ell_{1}^{2} \ell_{2}^{2}+\ell_{2}^{2} \ell_{3}^{2}+\ell_{3}^{2} \ell_{1}^{2}\right)-\ell_{1}^{4}-\ell_{2}^{4}-\ell_{3}^{4}}
$$

provides the area of a general triangle with side lengths $\ell_{1}, \ell_{2}$, and $\ell_{3}$. Combining Eqs. (29) and (30), and observing that the area per lattice site $A_{\text {site }}$ is twice the area of an elementary lattice triangle, i.e.,

$$
A_{\text {site }}=d_{1} h_{1}=2 A_{\mathrm{tr}}\left(d_{1}, d_{2}, d_{3}\right),
$$

we obtain, asymptotically close to $T_{\mathrm{c}}$, the relation

$$
V_{\text {co }}=\frac{2\left(1-z_{2} z_{3}\right)\left(z_{2}+z_{3}\right)}{\left(1-z_{2}^{2}\right)\left(1-z_{3}^{2}\right)}\left(\frac{\xi_{1}}{d_{1}}\right)^{2} A_{\text {site }},
$$

which will be needed below.

\section{Free energy}

In this section, we derive explicit results for the scaling behaviors of $f_{\mathrm{ex}}, f_{\mathrm{sf}}$, and $f_{\mathrm{fs}}$ for periodic and antiperiodic $\mathrm{bc}$ and of $f_{\mathrm{fs}}$ for free bc. We provide scaling functions for their contributions per bulk correlation volume, as well as standard scaling functions and critical Casimir amplitudes for them. The scaling functions and critical amplitudes will be expressed in terms of the scaling variable $\tilde{x}$ from (8) and the anisotropy parameters $r$ and $\theta$. For further reference we note that with (8) and (21) follows

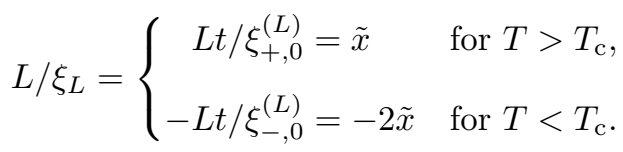

For the two-dimensional Ising model, the leading singular behavior of $f$ in the bulk limit $L \rightarrow \infty$ behaves as $\propto t^{2} \ln |t|$. The arbitrariness in splitting the constant under the logarithm between singular and nonsingular contributions to $f$ prevents the required unique splitting of $f$ according to (2) and causes a violation of scaling. Consequently, $\mathcal{F}$ does not exist. On the other hand, such a splitting of $f_{\mathrm{fs}}$ and therefore the function $\mathcal{G}$ exist for all cases considered in this work. The existence of a corresponding splitting for $f_{\mathrm{ex}}$ and $f_{\mathrm{sf}}$, and thus the existence of $\mathcal{A}$, depends on the bc, as we will see below.

It is useful to define the strip free energies per site $\hat{f}$ and per bulk correlation volume $\tilde{f}$ by

$$
\begin{aligned}
& \hat{f}(T, L)=A_{\text {site }} f(T, L), \\
& \tilde{f}(T, L)=V_{\text {co }} f(T, L),
\end{aligned}
$$


respectively. Let analogous definitions hold for $f_{\mathrm{b}}, f_{\mathrm{ex}}$, $f_{\mathrm{sf}}, f_{\mathrm{fs}}$, and, if a unique separation as in (2) is defined, also for their singular parts.

\section{Periodic and antiperiodic bc}

The free energy per site defined in (35a) is, according to Eq. (71) of Ref. [25], given by

$$
\begin{aligned}
\hat{f}(T, L) & =-C+\hat{f}_{\mathrm{sf}}(T, L) \\
& -\frac{1}{2 N} \sum_{j=0}^{N-1} \ln \frac{f_{1}\left(\phi_{j}\right)+\sqrt{f_{1}^{2}\left(\phi_{j}\right)-f_{2}^{2}\left(\phi_{j}\right)}}{2},
\end{aligned}
$$

with

$$
\begin{array}{ll}
\phi_{j} \equiv \frac{2 \pi\left(j+\frac{1}{2}\right)}{N}, & \text { for periodic bc, } \\
\phi_{j} \equiv \frac{2 \pi j}{N}, & \text { for antiperiodic bc }
\end{array}
$$

and where [26]

$$
\begin{aligned}
& f_{1}(\phi) \equiv A_{0}-A_{1} \cos \phi \\
& f_{2}(\phi) \equiv \sqrt{\left(A_{2}+A_{3}\right)^{2}-4 A_{2} A_{3} \sin ^{2}(\phi / 2)} \\
& A_{0} \equiv\left(1+z_{1}^{2}\right)\left(1+z_{2}^{2}\right)\left(1+z_{3}^{2}\right)+8 z_{1} z_{2} z_{3} \\
& A_{1} \equiv 2 z_{1}\left(1-z_{2}^{2}\right)\left(1-z_{3}^{2}\right) \\
& A_{2} \equiv 2 z_{2}\left(1-z_{3}^{2}\right)\left(1-z_{1}^{2}\right) \\
& A_{3} \equiv 2 z_{3}\left(1-z_{1}^{2}\right)\left(1-z_{2}^{2}\right)
\end{aligned}
$$

and

$$
C \equiv \ln \frac{2}{\sqrt{\left(1-z_{1}^{2}\right)\left(1-z_{2}^{2}\right)\left(1-z_{3}^{2}\right)}}
$$

with the qualification that the interface function $\hat{f}_{\mathrm{sf}}$ is missing in Ref. [25]. For $T>T_{\mathrm{c}}$ indeed $\hat{f}_{\mathrm{sf}}=0$. For finite $L$, there is no phase transition and therefore the free energy per site for $T<T_{\mathrm{c}}$ is obtained by analytic continuation of (36). While for periodic bc, this leaves $\hat{f}_{\text {sf }}$ at zero, the $j=0$ term has to be treated separately for antiperiodic bc and we obtain

$$
\begin{aligned}
& \hat{f}_{\mathrm{sf}}^{(\mathrm{p})}(T, L)=0 \\
& \hat{f}_{\mathrm{sf}}^{(\mathrm{a})}(T, L)=\frac{\Theta(-t)}{N} \ln \frac{\left(1-z_{1}\right)\left(1-z_{2} z_{3}\right)}{\left(1+z_{1}\right)\left(z_{2}+z_{3}\right)},
\end{aligned}
$$

with the Heaviside step function $\Theta$. The interface contribution for antiperiodic bc for $T<T_{\mathrm{c}}$ was overlooked in
Ref. [25], leading to erroneous results there for $T<T_{\mathrm{c}}$. In particular, the $\theta<0$ plots for $R=\infty$ in Fig. 3(a) and for $L_{y}=\infty$ in Fig. 3(b) are missing a linearly rising part towards $\theta \rightarrow-\infty$. This leads to incorrect statements between Eqs. (78) and (79) of Ref. [25] about the $\theta<0$ behavior of the infinitely long cylinder.

Taking the limit $L \rightarrow \infty$ of (36), we obtain the bulk free energy per site as

$$
\hat{f}_{\mathrm{b}}(T)=-C-\frac{1}{4 \pi} \int_{0}^{2 \pi} d \phi \ln \frac{f_{1}(\phi)+\sqrt{f_{1}^{2}(\phi)-f_{2}^{2}(\phi)}}{2}
$$

and we note that the critical point corresponds to

$$
A_{0}-A_{1}-A_{2}-A_{3}=0
$$

which is equivalent to $(26)$.

Combining (36) and (42) according to (5), we obtain in an obvious notation

$$
\begin{aligned}
\hat{f}_{\mathrm{fs}}(T, L)= & {\left[\frac{1}{4 \pi} \int_{0}^{2 \pi} d \phi-\frac{1}{2 N} \sum_{j=0}^{N-1}\right] \times } \\
& \ln \frac{f_{1}\left(\phi_{(j)}\right)+\sqrt{f_{1}^{2}\left(\phi_{(j)}\right)-f_{2}^{2}\left(\phi_{(j)}\right)}}{2} .
\end{aligned}
$$

From (38) we obtain

$$
\begin{aligned}
& f_{1}^{2}(\phi)-f_{2}^{2}(\phi)= \\
& \quad\left(A_{0}-A_{1}+A_{2}+A_{3}\right)\left(A_{0}-A_{1}-A_{2}-A_{3}\right) \\
& \quad+4\left[A_{0} A_{1}+A_{2} A_{3}-A_{1}^{2} \cos ^{2}(\phi / 2)\right] \sin ^{2}(\phi / 2) .
\end{aligned}
$$

Near $T_{\mathrm{c}}$ the quantity $A_{0}-A_{1}-A_{2}-A_{3}$ is small and an appropriate approximation for $0 \leq \phi \leq \pi$ may be written as

$$
\begin{aligned}
& \sqrt{f_{1}^{2}(\phi)-f_{2}^{2}(\phi)} \approx 2 \sqrt{A_{0} A_{1}+A_{2} A_{3}-A_{1}^{2} \cos ^{2} \frac{\phi}{2}} \sin \frac{\phi}{2} \\
& +\frac{\sqrt{\left(A_{0}-A_{1}\right) A_{1}+A_{2} A_{3}}}{N}\left(\sqrt{\tilde{x}^{2}+N^{2} \phi^{2}}-N \phi\right),
\end{aligned}
$$

where we have used that through linear order in $t$

$$
\begin{aligned}
& \pm N \sqrt{\frac{\left(A_{0}-A_{1}+A_{2}+A_{3}\right)\left(A_{0}-A_{1}-A_{2}-A_{3}\right)}{\left(A_{0}-A_{1}\right) A_{1}+A_{2} A_{3}}} \\
& \quad=L t / \xi_{+, 0}^{(1)}=\tilde{x} \quad \text { for } T \gtrless T_{\mathrm{c}},
\end{aligned}
$$

which follows from (27), (28), (34), and (39). An approximation analogous to (46) holds for $\pi \leq \phi \leq 2 \pi$, which is found by replacing $\phi \rightarrow 2 \pi-\phi$ there, under which (45) is invariant. Thus we may write 


$$
\begin{aligned}
\hat{f}_{\mathrm{fs}}(T, L) \approx & {\left[\frac{1}{4 \pi} \int_{0}^{2 \pi} d \phi-\frac{1}{2 N} \sum_{j=0}^{N-1}\right] \ln \frac{A_{0}-A_{1} \cos \phi_{(j)}+2 \sqrt{A_{0} A_{1}+A_{2} A_{3}-A_{1}^{2} \cos ^{2} \frac{\phi_{(j)}}{2}} \sin \frac{\phi_{(j)}}{2}}{2} } \\
& +\frac{\sqrt{\left(A_{0}-A_{1}\right) A_{1}+A_{2} A_{3}}}{\left(A_{0}-A_{1}\right) N}\left[\frac{1}{2 \pi} \int_{0}^{2 \pi} d \phi-\frac{1}{N} \sum_{j=0}^{N-1}\right]\left(\sqrt{\tilde{x}^{2}+N^{2} \phi_{(j)}^{2}}-N \phi_{(j)}\right) .
\end{aligned}
$$

Using the results (A1a) and (A2a) for periodic bc and (A1b) and (A2b) for antiperiodic bc, we obtain

$$
N^{2} \hat{f}_{\mathrm{fs}}^{(\mathrm{p} / \mathrm{a})}(T, L) \approx \frac{\sqrt{\left(A_{0}-A_{1}\right) A_{1}+A_{2} A_{3}}}{\left(A_{0}-A_{1}\right)} I^{(+/-)}(\tilde{x}),
$$

with

$$
I^{( \pm)}(x) \equiv-\frac{1}{\pi} \int_{0}^{\infty} d \omega \ln \left(1 \pm e^{-\sqrt{x^{2}+\omega^{2}}}\right),
$$

and where we note that

$$
I^{(+)}(0)=-\pi / 12, \quad I^{(-)}(0)=\pi / 6 .
$$

Close to the critical point, we obtain, after some algebra and with the help of (33),

$$
\frac{\sqrt{\left(A_{0}-A_{1}\right) A_{1}+A_{2} A_{3}}}{\left(A_{0}-A_{1}\right)} \approx\left(\frac{\xi_{1}}{d_{1}}\right)^{2} \frac{A_{\text {site }}}{V_{\text {co }}} .
$$

Combining this with (49) gives, close to $T_{\mathrm{c}}$,

$$
\hat{f}_{\mathrm{fs}}^{(\mathrm{p} / \mathrm{a})}(T, L) \approx \frac{\xi_{1}^{2}}{L^{2}} \frac{A_{\text {site }}}{V_{\mathrm{co}}} I^{(+/-)}(\tilde{x}) .
$$

Using (19a), (28), (33), and (34), we obtain for the interface contribution (41b) to the free energy per site for antiperiodic bc for temperatures close to $T_{\mathrm{c}}$

$$
\hat{f}_{\mathrm{sf}}^{(\mathrm{a})}(T, L) \approx-\frac{\xi_{1}^{2}}{L^{2}} \frac{A_{\text {site }}}{V_{\mathrm{co}}} \Theta(-\tilde{x}) \tilde{x} .
$$

For periodic and antiperiodic bc, the free energy contributions $f_{\mathrm{ex}}, f_{\mathrm{sf}}$, and $f_{\mathrm{fs}}$ are dominated by their leading singular parts $f_{\mathrm{ex}, \mathrm{s}}, f_{\mathrm{sf}, \mathrm{s}}$, and $f_{\mathrm{fs}, \mathrm{s}}$, respectively. With (19a), (34), and (35), we obtain the asymptotic singular finite-size and interface parts of the free energy per bulk correlation volume as

$$
\tilde{f}_{\mathrm{fs}, \mathrm{s}}^{(\mathrm{p} / \mathrm{a})}(t, L)= \begin{cases}I^{(+/-)}(\tilde{x}) / \tilde{x}^{2} & \text { for } T>T_{\mathrm{c}}, \\ I^{(+/-)}(\tilde{x}) /\left(4 \tilde{x}^{2}\right) & \text { for } T<T_{\mathrm{c}},\end{cases}
$$

and

$$
\begin{aligned}
& \tilde{f}_{\mathrm{sf}, \mathbf{s}}^{(\mathrm{p})}(t, L)=0, \\
& \tilde{f}_{\mathrm{sf}, \mathrm{s}}^{(\mathrm{a})}(t, L)=-\Theta(-\tilde{x}) /(4 \tilde{x}),
\end{aligned}
$$

respectively. With (5b) follows the asymptotic singular excess free energy per correlation volume

$$
\begin{aligned}
& \tilde{f}_{\mathrm{ex}, \mathrm{s}}^{(\mathrm{p})}(t, L)=\tilde{f}_{\mathrm{fs}, \mathrm{s}}^{(\mathrm{p})}(t, L), \\
& \tilde{f}_{\mathrm{ex}, \mathrm{s}}^{(\mathrm{a})}(t, L)= \begin{cases}\tilde{f}_{\mathrm{fs}, \mathrm{s}}^{(\mathrm{a})}(t, L) & \text { for } T>T_{\mathrm{c}}, \\
{\left[I^{(-)}(\tilde{x})-\tilde{x}\right] /\left(4 \tilde{x}^{2}\right)} & \text { for } T<T_{\mathrm{c}} .\end{cases}
\end{aligned}
$$

Eqs. (55)-(57) represent central results of the current section. Their most remarkable feature is that their right hand sides depend only on the scaling variable $\tilde{x}$ and not on the parameters $r$ and $\theta$ describing the anisotropy of the system at large distances (nor on any other details of how the anisotropy is realized). We conjecture that the right hand sides of (55)-(57) represent universal scaling functions in the bulk universality class of the two-dimensional Ising model. In Sec. III B 1, we will explain their independence of $r$ and $\theta$ in the more general context of $d$-dimensional films.

Next we derive standard free energy scaling functions and critical amplitudes. Combining (16) and (18b) gives

$$
V_{\mathrm{co}} / \xi_{L}^{2}=r \cos ^{2} \theta+r^{-1} \sin ^{2} \theta,
$$

so that multiplying (55) and (56) by $L^{2} / V_{\text {co }}$, while observing (34), gives asymptotically

$$
\begin{aligned}
L^{2} f_{\mathrm{fs}, \mathrm{s}}^{(\mathrm{p} / \mathrm{a})}(t, L) & =\mathcal{G}^{(\mathrm{p} / \mathrm{a})}(\tilde{x}, r, \theta) \\
& =\frac{I^{(+/-)}(\tilde{x})}{r \cos ^{2} \theta+r^{-1} \sin ^{2} \theta},
\end{aligned}
$$

and

$$
L^{2} f_{\mathrm{sf}, \mathrm{s}}^{(\mathrm{a})}(t, L)=-\frac{\Theta(-\tilde{x}) \tilde{x}}{r \cos ^{2} \theta+r^{-1} \sin ^{2} \theta},
$$

respectively. Therefore, we have

$$
\begin{aligned}
& L^{2} f_{\mathrm{ex}, \mathrm{s}}^{(\mathrm{p} / \mathrm{a})}(t, L)=\mathcal{A}^{(\mathrm{p} / \mathrm{a})}(\tilde{x}, r, \theta) \\
& \quad= \begin{cases}\mathcal{G}^{(\mathrm{p})}(\tilde{x}, r, \theta) & \text { for periodic bc, } \\
\frac{I^{(-)}(\tilde{x})-\Theta(-\tilde{x}) \tilde{x}}{r \cos ^{2} \theta+r^{-1} \sin ^{2} \theta} & \text { for antiperiodic bc, }\end{cases}
\end{aligned}
$$

where $\mathcal{A}^{(\mathrm{p} / \mathrm{a})}(\tilde{x}, r, \theta)$ and $\mathcal{G}^{(\mathrm{p} / \mathrm{a})}(\tilde{x}, r, \theta)$ are the scaling functions of the excess free energy density and the finitesize contribution to the free energy density, respectively. 


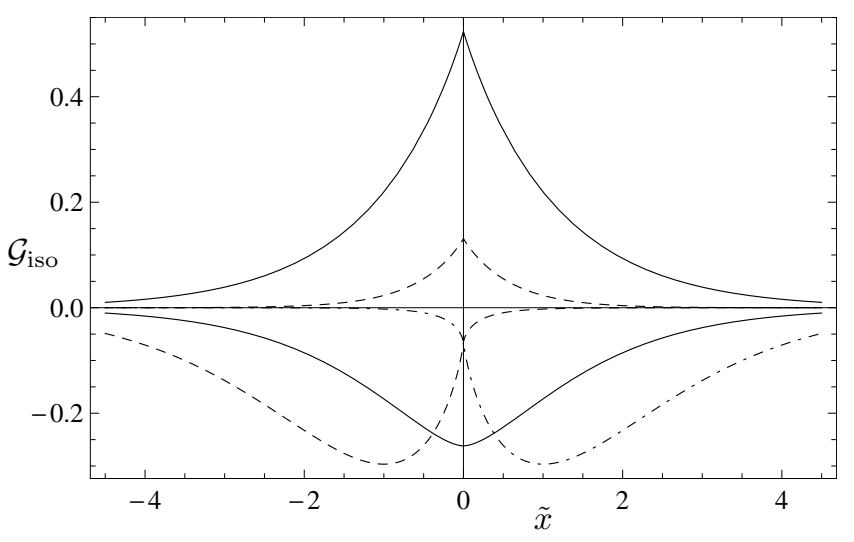

FIG. 3: Scaling functions $\mathcal{G}_{\text {iso }}^{(\mathrm{p})}(\tilde{x})($ solid, $<0), \mathcal{G}_{\text {iso }}^{(\mathrm{a})}(\tilde{x})$ (solid, $>0), \mathcal{G}_{\text {iso }}^{(00)}(\tilde{x})$ (dashed, $\left.<0\right), \mathcal{G}_{\text {iso }}^{(++)}(\tilde{x})$ (dot-dashed, $\left.<0\right)$, and $\mathcal{G}_{\text {iso }}^{(0+)}(\tilde{x})$ (dashed, $>0$ ).

For the two-dimensional Ising model, they generalize the isotopic-case structure (6) to the anisotropic case. We conjecture that $\mathcal{A}^{(\mathrm{p} / \mathrm{a})}$ and $\mathcal{G}^{(\mathrm{p} / \mathrm{a})}$ are universal functions of $\tilde{x}, r$, and $\theta$ for all infinite-strip systems in the bulk universality class of the two-dimensional Ising model with the appropriate bc in the $L$ direction. Note that the scaling functions depend on the anisotropy parameters $r$ and $\theta$ only through a geometric factor.

For the isotropic case $r=1$, the results (59) and (61) reduce to

$$
\mathcal{G}_{\text {iso }}^{(\mathrm{p} / \mathrm{a})}(\tilde{x})=I^{(+/-)}(\tilde{x})
$$

and

$$
\mathcal{A}_{\mathrm{iso}}^{(\mathrm{p} / \mathrm{a})}(\tilde{x})= \begin{cases}\mathcal{G}_{\mathrm{iso}}^{(\mathrm{p})}(\tilde{x}) & \text { for periodic bc } \\ I^{(-)}(\tilde{x})-\Theta(-\tilde{x}) \tilde{x} & \text { for antiperiodic bc }\end{cases}
$$

and thus we may relate

$$
\begin{aligned}
& \mathcal{G}^{(\mathrm{p} / \mathrm{a})}(\tilde{x}, r, \theta)=\left(r \cos ^{2} \theta+r^{-1} \sin ^{2} \theta\right)^{-1} \mathcal{G}_{\text {iso }}^{(\mathrm{p} / \mathrm{a})}(\tilde{x}), \\
& \mathcal{A}^{(\mathrm{p} / \mathrm{a})}(\tilde{x}, r, \theta)=\left(r \cos ^{2} \theta+r^{-1} \sin ^{2} \theta\right)^{-1} \mathcal{A}_{\mathrm{iso}}^{(\mathrm{p} / \mathrm{a})}(\tilde{x}) .
\end{aligned}
$$

With $x_{\perp}=\tilde{x}$, our result for $\mathcal{G}_{\text {iso }}^{(\mathrm{p})}(\tilde{x})$ is identical to $\Theta_{\perp}\left(x_{\perp}, 0\right)$ from Eq. (57) of Ref. [27]. Even for the isotropic limit, $\mathcal{G}^{(\mathrm{a})}$ and $\mathcal{A}^{(\mathrm{a})}$ appear not to have been previously published. $\mathcal{G}_{\text {iso }}^{(\mathrm{p})}(\tilde{x})$ and $\mathcal{G}_{\text {iso }}^{(\mathrm{a})}(\tilde{x})$ are shown in Fig. 3. A cross check of $\mathcal{G}_{\text {iso }}^{\text {(p/a) }}$ and $\mathcal{A}_{\text {iso }}^{(\mathrm{p} / \mathrm{a})}$ with published scaling functions for the Casimir force is provided in Appendix B 1 .

With (51), the critical Casimir amplitudes $\Delta(r, \theta)=$ $\mathcal{G}(0, r, \theta)$ corresponding to $(59)$ are

$$
\begin{aligned}
\Delta^{(\mathrm{p})}(r, \theta) & =\frac{-\pi / 12}{r \cos ^{2} \theta+r^{-1} \sin ^{2} \theta}, \\
\Delta^{(\mathrm{a})}(r, \theta) & =\frac{\pi / 6}{r \cos ^{2} \theta+r^{-1} \sin ^{2} \theta},
\end{aligned}
$$

which we expect to be universal functions of $r$ and $\theta$ within the bulk universality class of the two-dimensional Ising model.

\section{Free $b c$}

The free energy per site defined in (35a) is, according to Eq. (A15) of Ref. [23], for free bc given by [28]

$$
\begin{aligned}
& \hat{f}_{\text {rect }}^{(00)}\left(T, L_{\|}, L\right)=-\ln \left(2 \cosh K_{1} \cosh K_{2} \cosh K_{3}\right) \\
& +\frac{1}{N} \ln \left(\cosh K_{2} \cosh K_{3}\right)-\frac{1}{N_{\|}} \sum_{\vartheta} \ln \left|1-z_{1} e^{i \vartheta}\right| \\
& -\frac{1}{2 N_{\|} N} \sum_{\vartheta} \ln \left(p_{+} \lambda_{+}^{N-1}+p_{-} \lambda_{-}^{N-1}\right),
\end{aligned}
$$

with

$$
\begin{aligned}
\vartheta & =\frac{(2 p-1) \pi}{N_{\|}}, \quad p=1, \ldots, N_{\|}, \\
p_{ \pm} & = \pm \frac{A-\lambda_{\mp}-\bar{a}|E|}{\sqrt{(A-F)^{2}+4|E C|}}, \\
\lambda_{ \pm} & =\frac{A+F \pm \sqrt{(A-F)^{2}+4|E C|}}{2},
\end{aligned}
$$

and

$$
\begin{aligned}
\bar{a} & =-2 z_{1}|\sin \vartheta| /\left|1-z_{1} e^{i \vartheta}\right|^{2}, \\
b & =\left(1-z_{1}^{2}\right) /\left|1-z_{1} e^{i \vartheta}\right|^{2}, \\
A & =\left(\alpha^{2}+\eta^{2}+2 \alpha \eta \cos \vartheta\right) /\left|1-z_{1} e^{i \vartheta}\right|^{2}, \\
E & =(2 \beta \gamma \sin \vartheta) /\left|1-z_{1} e^{i \vartheta}\right|^{2}, \\
C & =(2 \alpha \eta \sin \vartheta) /\left|1-z_{1} e^{i \vartheta}\right|^{2}, \\
F & =\left(\beta^{2}+\gamma^{2}+2 \beta \gamma \cos \vartheta\right) /\left|1-z_{1} e^{i \vartheta}\right|^{2}, \\
\alpha & =z_{1}+z_{2} z_{3}, \\
\beta & =z_{2}+z_{3} z_{1}, \\
\gamma & =z_{3}+z_{1} z_{2}, \\
\eta & =-\left(1+z_{1} z_{2} z_{3}\right) .
\end{aligned}
$$

The strip free energy per site is obtained as

$$
\begin{aligned}
& \hat{f}^{(00)}(T, L)=\lim _{L_{\|} \rightarrow \infty} \hat{f}_{\text {rect }}^{(00)}\left(T, L_{\|}, L\right) \\
& =-\ln \left(2 \cosh K_{1} \cosh K_{2} \cosh K_{3}\right) \\
& \quad+\frac{1}{N} \ln \left(\cosh K_{2} \cosh K_{3}\right)-\frac{1}{2 \pi} \int_{0}^{2 \pi} d \vartheta \ln \left|1-z_{1} e^{i \vartheta}\right| \\
& \quad-\frac{1}{4 \pi N} \int_{0}^{2 \pi} d \vartheta \ln \left[\left(p_{+} \lambda_{+}^{N-1}+p_{-} \lambda_{-}^{N-1}\right)\right] .
\end{aligned}
$$

Taking the limit $L \rightarrow \infty$ and observing that $\lambda_{+}>\lambda_{-}$, we obtain the bulk free energy per site (42) in the alternative 
representation

$$
\begin{aligned}
& \hat{f}_{\mathrm{b}}(T)=\lim _{L \rightarrow \infty} \hat{f}^{(00)}(T, L) \\
& =-\ln \left(2 \cosh K_{1} \cosh K_{2} \cosh K_{3}\right) \\
& \quad-\frac{1}{2 \pi} \int_{0}^{2 \pi} d \vartheta \ln \left|1-z_{1} e^{i \vartheta}\right|-\frac{1}{4 \pi} \int_{0}^{2 \pi} d \vartheta \ln \lambda_{+} .
\end{aligned}
$$

Thus the excess free energy per site is

$$
\hat{f}_{\mathrm{ex}}^{(00)}(T, L)=\hat{f}_{\mathrm{sf}}^{(00)}(T, L)+\hat{f}_{\mathrm{fs}}^{(00)}(T, L),
$$

with

$$
\begin{aligned}
\hat{f}_{\mathrm{sf}}^{(00)}(T, L)= & \frac{1}{N} \ln \left(\cosh K_{2} \cosh K_{3}\right) \\
& -\frac{1}{4 \pi N} \int_{0}^{2 \pi} d \vartheta \ln \frac{p_{+}}{\lambda_{+}}
\end{aligned}
$$

and

$$
\begin{aligned}
& \hat{f}_{\mathrm{fs}}^{(00)}(T, L)= \\
& -\frac{1}{4 \pi N} \int_{0}^{2 \pi} d \vartheta \ln \left[1+\frac{p_{-}}{p_{+}}\left(\frac{\lambda_{-}}{\lambda_{+}}\right)^{N-1}\right],
\end{aligned}
$$

where we already anticipate that the latter expression does not contain any surface or interface terms. Near $T_{\mathrm{c}}$, dominant contributions to the integral in (74) arise only near $\vartheta=0$ and $\vartheta=2 \pi$. Since the integrand in (74) is invariant under $\vartheta \rightarrow 2 \pi-\vartheta$, it is sufficient to consider small positive $\vartheta$. Employing the small- $t$ expansion (28), we obtain

$$
\begin{aligned}
A+F & \approx 2\left(1-z_{2} z_{3}\right)^{2} \\
\sqrt{(A-F)^{2}+4|E C|} & \approx \frac{2\left(1-z_{2} z_{3}\right)^{2}}{N} \sqrt{\bar{x}^{2}+\omega^{2}}, \\
A-F-2 \bar{a}|E| & \approx \frac{2\left(1-z_{2} z_{3}\right)^{2}}{N} \bar{x}
\end{aligned}
$$

with

$$
\omega \equiv \frac{\left(1-z_{2}^{2}\right)\left(1-z_{3}^{2}\right)}{2\left(1-z_{2} z_{3}\right)\left(z_{2}+z_{3}\right)} N \vartheta
$$

and

$$
\begin{aligned}
\bar{x} & \equiv \frac{\left(1-z_{2}^{2}\right)\left(1-z_{3}^{2}\right)}{2\left(1-z_{2} z_{3}\right)\left(z_{2}+z_{3}\right)} \frac{N d_{1} t}{\xi_{+, 0}^{(1)}} \approx \frac{\xi_{1}^{2}}{V_{\mathrm{co}}} \frac{L t}{\xi_{+, 0}^{(1)}} \approx \frac{\xi_{\infty} \xi_{L}}{\xi_{>} \xi_{<}} \frac{L t}{\xi_{+, 0}^{(L)}} \\
& \approx\left[\left(r \sin ^{2} \theta+r^{-1} \cos ^{2} \theta\right)\left(r \cos ^{2} \theta+r^{-1} \sin ^{2} \theta\right)\right]^{-1 / 2} \tilde{x} \\
& =\left[1+\frac{1}{4}\left(r-r^{-1}\right)^{2} \sin ^{2}(2 \theta)\right]^{-1 / 2} \tilde{x},
\end{aligned}
$$

where (8), (10b), (15b), (17), (18), (19b), (21), (32), and (33) have been used. It follows that

$$
\begin{aligned}
& \lambda_{ \pm} \approx\left(1-z_{2} z_{3}\right)^{2}\left(1 \pm \frac{\sqrt{\bar{x}^{2}+\omega^{2}}}{N}\right), \\
& p_{ \pm} \approx \frac{\sqrt{\bar{x}^{2}+\omega^{2}} \pm \bar{x}}{2 \sqrt{\bar{x}^{2}+\omega^{2}}}
\end{aligned}
$$

and therefore that

$$
\begin{aligned}
\frac{p_{+}}{\lambda_{+}} & \approx \frac{1}{2\left(1-z_{2} z_{3}\right)^{2}}\left(1+\frac{\bar{x}}{\sqrt{\bar{x}^{2}+\omega^{2}}}\right), \\
\frac{p_{-}}{p_{+}} & \approx \frac{\sqrt{\bar{x}^{2}+\omega^{2}}-\bar{x}}{\sqrt{\bar{x}^{2}+\omega^{2}}+\bar{x}}, \\
\left(\frac{\lambda_{-}}{\lambda_{+}}\right)^{N-1} & \approx e^{-2 \sqrt{\bar{x}^{2}+\omega^{2}}}
\end{aligned}
$$

From the expression for $p_{+} / \lambda_{+}$we conclude that the second term on the right hand side of (73) receives a nonsingular contribution $N^{-1}\left[\ln \left(1-z_{2} z_{3}\right)-\frac{1}{2} \ln 2\right]$ plus a contribution to the integral that is not restricted to small $\vartheta$ or $2 \pi-\vartheta$, since $\ln \left(1+\bar{x} / \sqrt{\bar{x}^{2}+\omega^{2}}\right) \approx \bar{x} / \omega$ for large $\omega$. This points towards a singular contribution that logarithmically violates scaling of $f_{\mathrm{sf}}^{(00)}$, and we will not consider this quantity in what follows.

Combining (74), (76), and (79), we obtain in the large$N$ limit close to $T_{\mathrm{c}}$ for the finite-size term

$$
N^{2} \hat{f}_{\mathrm{fs}}^{(00)}(T, L) \approx \frac{2\left(1-z_{2} z_{3}\right)\left(z_{2}+z_{3}\right)}{\left(1-z_{2}^{2}\right)\left(1-z_{3}^{2}\right)} I^{(f)}(\bar{x}),
$$

with

$$
\begin{aligned}
& I^{(f)}(x) \\
& \equiv-\frac{1}{2 \pi} \int_{0}^{\infty} d \omega \ln \left(1+\frac{\sqrt{x^{2}+\omega^{2}}-x}{\sqrt{x^{2}+\omega^{2}}+\bar{x}} e^{-2 \sqrt{x^{2}+\omega^{2}}}\right) .
\end{aligned}
$$

We note that

$$
I^{(f)}(0)=I^{(+)}(0) / 4=-\pi / 48,
$$

which results from comparing (82) with (50) and (51).

Combining (10b), (15b), (16), (19b), (32), (33), and (80), we obtain, close to $T_{\mathrm{c}}$,

$$
\hat{f}_{\mathrm{fs}}^{(00)}(T, L) \approx \frac{\xi_{>}^{2} \xi_{<}^{2}}{L^{2} \xi_{\infty}^{2}} \frac{A_{\text {site }}}{V_{\mathrm{co}}} I^{(f)}(\bar{x}) .
$$

For free bc, the free energy contribution $f_{\mathrm{fs}}$ is dominated by its leading singular part $f_{\mathrm{fs}, \mathrm{s}}$. With (1), (21), (35), and (77), we obtain for the asymptotic singular finitesize part of the free energy per bulk correlation volume

$$
\tilde{f}_{\mathrm{fs}, \mathrm{s}}^{(00)}(t, L)= \begin{cases}I^{(f)}(\bar{x}) / \bar{x}^{2} & \text { for } T>T_{\mathrm{c}}, \\ I^{(f)}(\bar{x}) /\left(4 \bar{x}^{2}\right) & \text { for } T<T_{\mathrm{c}}\end{cases}
$$

where Eq. (77) gives $\bar{x}$ as a function of $\tilde{x}, r$, and $\theta$. Eq. (84) represents a central result of the current section. Its most remarkable feature is that its right hand side depends only on the single variable $\bar{x}$. We conjecture that the right hand side of (84) represents a universal scaling function in the bulk universality class of the twodimensional Ising model. In Sec. III B 2, we will explain its independence of $r$ and $\theta$ in the more general context of $d$-dimensional films. 
Next we derive standard free energy scaling functions and critical amplitudes. Combining (16) and (18a) gives, in the asymptotic critical domain,

$$
V_{\mathrm{co}} / \xi_{\infty}^{2}=r \sin ^{2} \theta+r^{-1} \cos ^{2} \theta,
$$

so that, multiplying (84) by $L^{2} / V_{\text {co }}$ while observing (1), (21), (77), and (85), gives asymptotically

$$
\begin{aligned}
L^{2} f_{\mathrm{fs}, \mathrm{s}}^{(00)}(t, L) & =\mathcal{G}^{(00)}(\tilde{x}, r, \theta) \\
& =\left(r \sin ^{2} \theta+r^{-1} \cos ^{2} \theta\right) I^{(f)}(\bar{x}),
\end{aligned}
$$

with $\bar{x}$ from (77). We conjecture that $\mathcal{G}^{(00)}$ is a universal function of $\tilde{x}, r$, and $\theta$ in the bulk universality class of the two-dimensional Ising model. Note that the scaling function $\mathcal{G}^{(00)}$ depends on the anisotropy parameters $r$ and $\theta$ only through the geometric factors on the right hand sides of (77) and (86).

With (82), the related Casimir amplitude is

$$
\begin{aligned}
\Delta^{(00)}(r, \theta) & =\mathcal{G}^{(00)}(0, r, \theta) \\
& =-\frac{\pi}{48}\left(r \sin ^{2} \theta+r^{-1} \cos ^{2} \theta\right) .
\end{aligned}
$$

For the isotropic case, Eq. (86) reduces to

$$
\mathcal{G}_{\text {iso }}^{(00)}(\tilde{x})=I^{(f)}(\tilde{x}),
$$

and thus we may relate

$$
\mathcal{G}^{(00)}(\tilde{x}, r, \theta)=\left(r \sin ^{2} \theta+r^{-1} \cos ^{2} \theta\right) \mathcal{G}_{\text {iso }}^{(00)}(\bar{x}),
$$

with $\bar{x}$ from $(77)$. With $x=2 \tilde{x}$, our result for $\mathcal{G}_{\text {iso }}^{(00)}(\tilde{x})$ is identical to $X_{\mathrm{ex}}^{(o, o)}(x)$ for ordinary bc provided at the beginning of Sec. 12.1.2 in [9] without derivation. $\mathcal{G}_{\text {iso }}^{(00)}(\tilde{x})$ is shown in Fig. 3. A derivation of $\mathcal{G}_{\text {iso }}^{(00)}$ from published scaling functions for the Casimir force is provided in Appendix B 2.

As another cross check, we compare our results with the Casimir amplitude found in Ref. [23], where it is defined by

$$
\Delta_{\mathrm{INW}} \equiv N^{2} \hat{f}_{\mathrm{fs}, \mathrm{s}}(0, L),
$$

which, observing (10b), (15b), and (32), is related to $\Delta(r, \theta)=\mathcal{G}(0, r, \theta)$ by

$$
\Delta_{\mathrm{INW}}=\left(d_{1} / h_{1}\right) \Delta(r, \theta) .
$$

Ref. [23] provides the result

$$
\Delta_{\text {INW }}^{(00)}=-(\pi / 48) \eta,
$$

with

$$
\eta \equiv \frac{C_{2}^{2}}{S_{1}+S_{2}}=\frac{d_{1}}{h_{1}}\left(r \sin ^{2} \theta+r^{-1} \cos ^{2} \theta\right),
$$

where $C_{i} \equiv \cosh \left(2 K_{i}\right)$ and $S_{i} \equiv \sinh \left(2 K_{i}\right)$. The second equality in (93) is obtained by using (16), (17), (18b), (19b), (25), (32), and (33), as well as the criticality condition

$$
S_{1} S_{2}+S_{2} S_{3}+S_{3} S_{1}=1
$$

which is equivalent to (26) and (43). Comparison of (87) and (91)-(93) shows that our result for $\Delta^{(00)}(r, \theta)$ agrees with the corresponding result in Ref. [23]. However, while $\Delta^{(00)}(r, \theta)$ is universal, this is not the case for $\Delta_{\text {INW }}$ as defined in (90), since $\Delta_{\text {INW }}$ depends on lattice details as manifested by the factor $d_{1} / h_{1}$ appearing in (91) and (93).

\section{FILM IN $d$ DIMENSIONS}

The results of Sec. II may be analyzed in the more general context of a $d$-dimensional film of thickness $L$, i.e., for a system in an $L \times \infty^{d-1}$ geometry near a $d$ dimensional bulk critical point. Note that, within this section, we refer without further mentioning always to the asymptotic critical domain.

\section{A. Bulk correlation lengths}

Weakly anisotropic systems may be related to corresponding isotropic systems by an anisotropic scale transformation, see, e.g., [29]. Such a transformation may be realized as a shear transformation (see $[16,18,19]$ and [17] for the use of such a transformation in the context of critical phenomena for field-theoretic models and for $\varphi^{4}$ lattice models, respectively)

$$
\mathbf{x}^{\prime}=\mathbf{M x}
$$

where $\mathbf{x}$ and $\mathbf{x}^{\prime}$ represent the $d$ Cartesian coordinates of position vectors in the original anisotropic system and the related isotropic system, respectively, and where the $d \times d$ matrix $\mathbf{M}$ may be decomposed according to

$$
\mathbf{M}=\mathbf{S R}
$$

into a rotation, represented by an orthogonal matrix $\mathbf{R}$, and a subsequent rescaling, represented by a real diagonal matrix $\mathbf{S}$.

It is then straightforward to adapt the above transformation to the case treated here, where the long-distance correlations of the anisotropic bulk system are described by a correlation lengths ellipsoid represented by a tensor $\boldsymbol{\Xi}$ that is diagonalized by $\mathbf{R}$, so that

$$
\mathbf{R} \Xi \mathbf{R}^{-1}=\operatorname{diag}\left(\xi_{1}^{2}, \xi_{2}^{2}, \ldots, \xi_{d}^{2}\right),
$$

with the correlation lengths $\xi_{1}, \ldots, \xi_{d}$ along the principal axes of the ellipsoid, and where $\mathbf{S}$ is the volumeconserving (det $\mathbf{S}=1$ ) rescaling matrix

$$
\mathbf{S}=V_{\mathrm{co}}^{1 / d} \operatorname{diag}\left(\xi_{1}^{-1}, \xi_{2}^{-1}, \ldots, \xi_{d}^{-1}\right),
$$


with the correlation volume defined by

$$
V_{\text {co }} \equiv \xi_{1} \xi_{2} \cdots \xi_{d}=(\operatorname{det} \boldsymbol{\Xi})^{1 / 2} .
$$

Thus the correlation length in the isotropic system is

$$
\xi=V_{\mathrm{co}}^{1 / d}
$$

Consequently, the squared correlation lengths tensor in the primed system is

$$
\boldsymbol{\Xi}^{\prime}=\mathbf{M} \boldsymbol{\Xi} \mathbf{M}^{T}=V_{\mathrm{co}}^{2 / d} \mathbb{1}
$$

with the $d \times d$ unit matrix $\mathbb{1}$. Note that

$$
\Xi^{-1}=V_{\mathrm{co}}^{-2 / d} \mathbf{M}^{T} \mathbf{M}
$$

In the $\varphi^{4}$ lattice model and in the field-theoretic contexts of Refs. [17] and [16, 18], respectively, the shear transformation is written as $\boldsymbol{\lambda}^{-1 / 2} \mathbf{U}$, where $\mathbf{U}$ corresponds to our rotation $\mathbf{R}$ and $\boldsymbol{\lambda}^{-1 / 2}$ corresponds to $\mathbf{S}$ with the qualification that, in contrast to our definition, $\boldsymbol{\lambda}$ is not necessarily volume-conserving. In Refs. [1618], a matrix $\mathbf{A}=\mathbf{U}^{-1} \boldsymbol{\lambda} \mathbf{U}$ was defined, so that $\overline{\mathbf{A}} \equiv$ $\mathbf{A} /(\operatorname{det} \mathbf{A})^{1 / d}$ may be used to parameterize the longdistance anisotropy [18]. Since we choose $\mathbf{S}$ to be volumeconserving, our conventions imply

$$
\mathbf{A}=\overline{\mathbf{A}}=\left(\mathbf{M}^{T} \mathbf{M}\right)^{-1}=(\operatorname{det} \boldsymbol{\Xi})^{-1 / d} \boldsymbol{\Xi},
$$

with

$$
\operatorname{det} \mathbf{A}=\operatorname{det} \overline{\mathbf{A}}=1
$$

Note that $\overline{\mathbf{A}}$ is defined here through the physical correlation lengths in the asymptotic critical domain. In contrast, explicit versions of $\overline{\mathbf{A}}$ were obtained in [16-18] by requiring the related shear transformation to lead to a transformed Hamiltonian, whose expansion in small wave numbers $k$ is isotropic through order $k^{2}$. While for standard $\varphi^{4}$ field theory these definitions should coincide due to an exact mapping between the anisotropic and isotropic bulk Hamiltonians [16], there is no reason to believe that this procedure generally leads also for lattice models to $\overline{\mathbf{A}}$ as defined here. We will return to this issue in Sec. IV.

Let $\hat{\mathbf{n}}$ be a unit vector that is orthogonal to the film boundaries and define the vector

$$
\mathbf{L}=L \hat{\mathbf{n}} \text {. }
$$

For two-dimensional systems such as the Ising model treated in Sec. II, it is convenient to choose coordinates, where

$$
\hat{\mathbf{n}}^{T}=(1,0),
$$

so that, with $r$ and $\theta$ as defined in Sec. II B, we have

$$
\mathbf{R}=\left(\begin{array}{cc}
\cos \theta & \sin \theta \\
-\sin \theta & \cos \theta
\end{array}\right)
$$

and

$$
\mathbf{S}=\left(\begin{array}{cc}
r^{1 / 2} & 0 \\
0 & r^{-1 / 2}
\end{array}\right)
$$

from which follows with (96) and (103)

$$
\overline{\mathbf{A}}^{-1}=\left(\begin{array}{cc}
r \cos ^{2} \theta+r^{-1} \sin ^{2} \theta & \frac{1}{2}\left(r-r^{-1}\right) \sin (2 \theta) \\
\frac{1}{2}\left(r-r^{-1}\right) \sin (2 \theta) & r \sin ^{2} \theta+r^{-1} \cos ^{2} \theta
\end{array}\right) .
$$

Therefore, the parameterization of the anisotropy of a $d$-dimensional system by $\overline{\mathbf{A}}$ and the corresponding film orientation by $\hat{\mathbf{n}}$ reduces, for a two-dimensional system, naturally to the parameterization by $r$ and $\theta$, as manifested, for the two-dimensional Ising model, by Eqs. (59)(61), (65), (86), and (87).

\section{B. Free energy}

For a $d$-dimensional system with restricted geometry, the system shape and the bc are generally transformed in a nontrivial way by the shear transformation. A major simplification arises for films with (anti-)periodic, fixed or free bc, since (i) a film is transformed into another film and (ii) the bc are invariant. This means that we can express film free energy scaling functions of the anisotropic system in terms of the corresponding scaling functions of the isotropic system with the same geometry and bc. Then the modifications for the anisotropic system can be represented by geometric factors, as we already explicitly observed in Sec. II for the two-dimensional Ising model and as will be detailed for $d$-dimensional film systems below.

For notational simplicity, we formulate what follows for the singular part $f_{\mathrm{s}}$ of the free energy density $f$, even though for the particular case of the two-dimensional Ising model there is no unique separation of regular part $f_{\mathrm{s}}$ and singular part $f_{\mathrm{ns}}$ and consequently $\mathcal{F}$ does not exist, in contrast to $\mathcal{A}$ and/or $\mathcal{G}$, depending on the bc. If universality continues to hold for anisotropic $d$ dimensional film systems, we expect Eq. (3) to be replaced by

$$
L^{d} f_{s}(t, L)=\mathcal{F}(\tilde{x}, \overline{\mathbf{A}}, \hat{\mathbf{n}}),
$$

where $\mathcal{F}$ is a universal function of its arguments and the imposed bc. Eqs. (6) are replaced analogously.

Due to the different ways a film transforms for periodic and antiperiodic bc on the one hand and for fixed and free bc on the other hand, we treat these cases separately.

\section{Periodic and antiperiodic $b c$}

Consider a film with periodic or antiperiodic bc and a length $L$ of (anti-)periodicity in the direction represented 


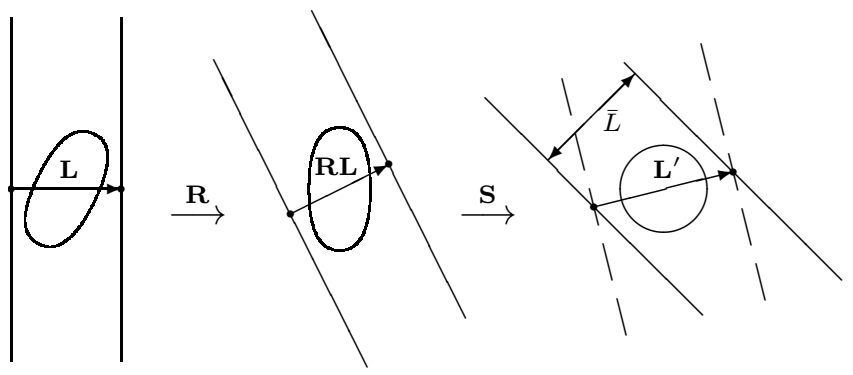

FIG. 4: Illustration of the shear transformation from an anisotropic to an isotropic film for $d=2$, i.e., for an infinite strip. Shown are the behavior of the strip boundaries, the correlation ellipse, and the vector $\mathbf{L}$ under a rotation $\mathbf{R}$ and a subsequent rescaling $\mathbf{S}$ along the horizontal and vertical coordinate axes. $\bar{L}$ is the thickness of the resulting strip, needed for free and fixed bc. For periodic and antiperiodic bc, the indicated dots at opposite sides of the original, rotated, and rescaled strips, connected by the vectors $\mathbf{L}, \mathbf{R L}$, and $\mathbf{L}^{\prime}=\mathbf{S R L}$, respectively, are physically identical. For these bc, translational invariance allows to define new strip boundaries (dashed) that are perpendicular to the direction of (anti-)periodicity, so that $L^{\prime}=\left|\mathbf{L}^{\prime}\right|$ is the thickness of the resulting strip.

by the unit vector $\hat{\mathbf{n}}$. The shear transformation $\mathbf{M}$ transforms the film into another film, albeit with a different vector

$$
\mathbf{L}^{\prime}=\mathbf{M L}
$$

describing the direction and length $L^{\prime}=\left|\mathbf{L}^{\prime}\right|$ of (anti-)periodicity of the isotropic system. For (anti-)periodic bc, the orientation of the film boundaries is not unique. We choose them such that $\mathbf{L}$ is orthogonal to the boundaries of the original film and $\mathbf{L}^{\prime}$ is orthogonal to the boundaries of the film with isotropic bulk correlation lengths, see Fig. 4. Other choices lead to skewed bc. Results for such bc are related to the results presented here by elementary geometric considerations.

Now consider singular parts of free energy densities that exhibit scaling. For the isotropic model, we assume

$$
L^{\prime d} f_{\text {iso }, \mathrm{s}}\left(t, L^{\prime}\right)=\mathcal{F}_{\text {iso }}\left(\tilde{x}^{\prime}\right),
$$

with the scaling variable $\tilde{x}^{\prime} \equiv\left(L^{\prime} / \xi_{+, 0}\right)^{1 / \nu} t$. With the critical behavior (9) of the correlation length $\xi_{L}$ in the direction $\mathbf{L}$ of (anti-)periodicity of the original system, the scaling variables $\tilde{x}$ from (8) and $\tilde{x}^{\prime}$ from above are identical, since with (101) and (111) we have

$$
\left(L / \xi_{L}\right)^{2}=\mathbf{L}^{T} \boldsymbol{\Xi}^{-1} \mathbf{L}=\mathbf{L}^{\prime T} \boldsymbol{\Xi}^{\prime-1} \mathbf{L}^{\prime}=\left(L^{\prime} / \xi\right)^{2} .
$$

Therefore, we may write for the free energy per correlation volume

$$
\begin{aligned}
\tilde{f}_{\mathrm{s}}(t, L) & =V_{\mathrm{co}} f_{\mathrm{s}}(t, L)=V_{\mathrm{co}} f_{\mathrm{iso}, \mathrm{s}}\left(t, L^{\prime}\right) \\
& = \begin{cases}\tilde{x}^{-d \nu} \mathcal{F}_{\text {iso }}(\tilde{x}) & \text { for } T>T_{\mathrm{c}}, \\
R_{\xi}^{-d}(-\tilde{x})^{-d \nu} \mathcal{F}_{\text {iso }}(\tilde{x}) & \text { for } T<T_{\mathrm{c}},\end{cases}
\end{aligned}
$$

where we have used Eq. (100) and that our shear transformation is volume-conserving and thus separately conserves the correlation volume and the free energy density. Analogous equations hold for $f_{\mathrm{ex}, \mathrm{s}}, f_{\mathrm{sf}, \mathrm{s}}$, and $f_{\mathrm{fs}, \mathrm{s}}$, if they exhibit scaling. Thus we find for $d$-dimensional films with periodic or antiperiodic bc that the leading singular parts of (scaling) free energy densities per correlation volume depend only on the ratio of the length of (anti-)periodicity and the correlation length in the corresponding direction and not on any other details of the shape or orientation of the correlation ellipsoid. For the two-dimensional Ising model this is reflected by the explicit results (55)-(57).

Multiplying (114) by

$$
L^{d} / V_{\mathrm{co}}=\left(L / L^{\prime}\right)^{d} \times \begin{cases}\tilde{x}^{d \nu} & \text { for } T>T_{\mathrm{c}} \\ R_{\xi}^{d}(-\tilde{x})^{d \nu} & \text { for } T<T_{\mathrm{c}}\end{cases}
$$

and observing

$$
L^{\prime}=L|\mathbf{M} \hat{\mathbf{n}}|=L\left(\hat{\mathbf{n}}^{T} \overline{\mathbf{A}}^{-1} \hat{\mathbf{n}}\right)^{1 / 2},
$$

which follows from (103), (105), and (111), we obtain the standard free energy scaling function

$$
\begin{aligned}
L^{d} f_{\mathrm{s}}(t, L) & =\mathcal{F}(\tilde{x}, \overline{\mathbf{A}}, \hat{\mathbf{n}}) \\
& =\left(\hat{\mathbf{n}}^{T} \overline{\mathbf{A}}^{-1} \hat{\mathbf{n}}\right)^{-d / 2} \mathcal{F}_{\text {iso }}(\tilde{x}) .
\end{aligned}
$$

That is, $\mathcal{F}$ depends only through a geometric factor on the relative orientations of the direction of (anti-)periodicity and the correlation ellipsoid.

Specializing to $d=2-$ dimensional systems with conventions as in (106)-(109), Eq. (117) reads

$$
\begin{aligned}
L^{2} f_{\mathrm{s}}(t, L) & =\mathcal{F}(\tilde{x}, r, \theta) \\
& =\left(r \cos ^{2} \theta+r^{-1} \sin ^{2} \theta\right)^{-1} \mathcal{F}_{\text {iso }}(\tilde{x}) .
\end{aligned}
$$

Analogous equations hold for $f_{\mathrm{ex}, \mathrm{s}}, f_{\mathrm{fs}, \mathrm{s}}$, and $f_{\mathrm{sf}, \mathrm{s}}$, if they exhibit scaling. This explains, from a more general point of view, the anisotropy dependence of the Ising model scaling functions presented in Eqs. (59)-(64).

\section{Fixed and free bc}

Here we repeat the considerations of the preceding section for a film with fixed and/or free bc, such as $00,++$, $0+$, or +- bc. The treatment immediately extends to similar other invariant bc. For definiteness, we choose coordinates where the " 1 " direction is normal to the film boundaries, i.e., where

$$
\hat{\mathbf{n}}^{T}=(1,0, \ldots, 0) .
$$

As opposed to the case of periodic or antiperiodic bc, the thickness of the isotropic film is no longer given by the length of the transformed vector $\mathbf{L}^{\prime}$, see Fig 4 . Instead, 
according to Eq. (2.48) of Ref. [30], the film thickness $\bar{L}$ of the isotropic system is given by

$$
\bar{L}=\left(\operatorname{det} \overline{\mathbf{A}}^{-1} / \operatorname{det}\left[\left[\overline{\mathbf{A}}^{-1}\right]\right]\right)^{1 / 2} L,
$$

where $\left[\left[\overline{\mathbf{A}}^{-1}\right]\right]$ is the $(d-1) \times(d-1)$ right lower part of $\overline{\mathbf{A}}^{-1}$ and where, with the conventions employed here, $\operatorname{det} \overline{\mathbf{A}}^{-1}=1$, compare Eq. (104).

For the isotropic model, we assume

$$
\bar{L}^{d} f_{\text {iso }, \mathrm{s}}(t, \bar{L})=\mathcal{F}_{\text {iso }}(\bar{x}),
$$

with the scaling variable $\bar{x} \equiv\left(\bar{L} / \xi_{+, 0}\right)^{1 / \nu} t$. From (100), (103)-(105), (119), and (120), we obtain

$$
\left(L / \xi_{L}\right)^{2}=\mathbf{L}^{T} \boldsymbol{\Xi}^{-1} \mathbf{L}=\left(\overline{\mathbf{A}}^{-1}\right)_{11} \operatorname{det}\left[\left[\overline{\mathbf{A}}^{-1}\right]\right](\bar{L} / \xi)^{2} .
$$

With $\bar{x}$ from above and $\tilde{x}$ from (8) this translates to the relation

$$
\bar{x}=\left\{\left(\overline{\mathbf{A}}^{-1}\right)_{11} \operatorname{det}\left[\left[\overline{\mathbf{A}}^{-1}\right]\right]\right\}^{-1 /(2 \nu)} \tilde{x} .
$$

For the singular part of the free energy per correlation volume, we may write

$$
\begin{aligned}
\tilde{f}_{\mathrm{s}}(t, L) & =V_{\mathrm{co}} f_{\mathrm{s}}(t, L)=V_{\mathrm{co}} f_{\mathrm{iso}, \mathrm{s}}(t, \bar{L}) \\
& = \begin{cases}\bar{x}^{-d \nu} \mathcal{F}_{\mathrm{iso}}(\bar{x}) & \text { for } T>T_{\mathrm{c}} \\
R_{\xi}^{-d}(-\bar{x})^{-d \nu} \mathcal{F}_{\mathrm{iso}}(\bar{x}) & \text { for } T<T_{\mathrm{c}}\end{cases}
\end{aligned}
$$

where we have used Eq. (100) and that our shear transformation is volume-conserving and thus separately conserves the correlation volume and the free energy density. Analogous equations hold for $f_{\mathrm{ex}, \mathrm{s}}, f_{\mathrm{sf}, \mathrm{s}}$, and $f_{\mathrm{fs}, \mathrm{s}}$, if they exhibit scaling. Note that as for the case of (anti-)periodic bc, the free energy per correlation volume depends only on one suitably chosen scaling variable. For the two-dimensional Ising model with free bc, this is reflected by the explicit result (84).

Multiplying (124) by

$$
L^{d} / V_{\mathrm{co}}=(L / \bar{L})^{d} \times \begin{cases}\bar{x}^{d \nu} & \text { for } T>T_{\mathrm{c}}, \\ R_{\xi}^{d}(-\bar{x})^{d \nu} & \text { for } T<T_{\mathrm{c}}\end{cases}
$$

and observing (104) and (120), we obtain the standard free energy scaling function

$$
\begin{aligned}
L^{d} f_{\mathrm{s}}(t, L) & =\mathcal{F}(\tilde{x}, \overline{\mathbf{A}}, \hat{\mathbf{n}}) \\
& =\left(\operatorname{det}\left[\left[\overline{\mathbf{A}}^{-1}\right]\right]\right)^{d / 2} \mathcal{F}_{\text {iso }}(\bar{x}),
\end{aligned}
$$

with $\bar{x}$ from (123). Note that the right hand sides of Eqs. (120), (122), (123), and (126) change their form for a choice of coordinates that does not imply Eq. (119). The general form may be obtained by replacing

$$
\begin{aligned}
\left(\overline{\mathbf{A}}^{-1}\right)_{11} & \rightarrow \hat{\mathbf{n}}^{T} \overline{\mathbf{A}}^{-1} \hat{\mathbf{n}}, \\
\operatorname{det}\left[\left[\overline{\mathbf{A}}^{-1}\right]\right] & \rightarrow-\frac{\operatorname{det}\left(\overline{\mathbf{A}}^{-1}-\hat{\mathbf{n}} \hat{\mathbf{n}}^{T} \overline{\mathbf{A}}^{-1}-\overline{\mathbf{A}}^{-1} \hat{\mathbf{n}} \hat{\mathbf{n}}^{T}\right)}{\hat{\mathbf{n}}^{T} \overline{\mathbf{A}}^{-1} \hat{\mathbf{n}}} .
\end{aligned}
$$

Specializing to $d=2$-dimensional systems with conventions as in (106)-(109), Eq. (126) reads

$$
\begin{aligned}
L^{2} f_{\mathrm{s}}(t, L) & =\mathcal{F}(\tilde{x}, r, \theta) \\
& =\left(r \sin ^{2} \theta+r^{-1} \cos ^{2} \theta\right) \mathcal{F}_{\text {iso }}(\bar{x}),
\end{aligned}
$$

with

$$
\bar{x}=\left[1+\frac{1}{4}\left(r-r^{-1}\right)^{2} \sin ^{2}(2 \theta)\right]^{-1 /(2 \nu)} \tilde{x},
$$

as obtained by combining $\left(\overline{\mathbf{A}}^{-1}\right)_{11}$ and $\operatorname{det}\left(\left[\left[\overline{\mathbf{A}}^{-1}\right]\right]\right)=$ $\left(\overline{\mathbf{A}}^{-1}\right)_{22}$ from (109) with (123). With $\nu$ from $(21)$, Eq. (129) reduces to the relation (77) found for the twodimensional Ising model. Equations analogous to (128) hold for $f_{\mathrm{ex}, \mathrm{s}}, f_{\mathrm{sf}, \mathrm{s}}$, and $f_{\mathrm{fs}, \mathrm{s}}$, if they exhibit scaling. As for periodic and antiperiodic bc, the $d$-dimensional point of view provides an explanation for the anisotropy dependence of the Ising model scaling function for free bc presented in (89).

In Appendix B2, we sketch the derivation of the isotropic Ising model results

$$
\begin{aligned}
\mathcal{G}_{\text {iso }}^{(++)}(\tilde{x}) & =\mathcal{G}_{\text {iso }}^{(00)}(-\tilde{x})=I^{(f)}(-\tilde{x}), \\
\mathcal{G}_{\text {iso }}^{(0+)}(\tilde{x}) & =\mathcal{G}_{\text {iso }}^{(\mathrm{a})}(2 \tilde{x}) / 4=I^{(-)}(2 \tilde{x}) / 4,
\end{aligned}
$$

with $I^{(f)}$ and $I^{(-)}$from (81) and (50), respectively. With $x=2 \tilde{x}$, our result for $\mathcal{G}_{\text {iso }}^{(++)}(\tilde{x})$ is identical to $X_{\text {ex }}^{(+,+)}(x)$ provided at the beginning of Sec. 12.1.2 in [9] without derivation. $\mathcal{G}_{\text {iso }}^{(++)}(\tilde{x})$ and $\mathcal{G}_{\text {iso }}^{(0+)}(\tilde{x})$ are shown in Fig. 3. Together with (77), (124), and (128), we immediately obtain predictions for both the finite-size scaling behavior of the finite-size part of the free energy per correlation volume and the scaling functions of the finite-size part of the free energy for the anisotropic case for both ++ and $0+$ bc. Combining (128) with (130), while observing (51) and (82), the related Casimir amplitudes are

$$
\Delta^{(++)}(r, \theta)=-\Delta^{(0+)}(r, \theta) / 2=\Delta^{(00)}(r, \theta),
$$

with $\Delta^{(00)}(r, \theta)$ from (87). With the definition (90), the results provided at the end of Sec. 2 in Ref. [23] for the Casimir amplitudes are, for,$++ 0+$, and $00 \mathrm{bc}$,

$$
\Delta_{\mathrm{INW}}^{(++)}=-\Delta_{\mathrm{INW}}^{(0+)} / 2=\Delta_{\mathrm{INW}}^{(00)}
$$

with $\Delta_{\text {INW }}^{(00)}$ from (92). While the results for $\Delta_{\text {INW }}^{(++)}$and $\Delta_{\text {INW }}^{(0+)}$ were derived in Ref. [23] only for rectangular lattices, the combination of (91), (131), and (132) shows that they remain correct for the general triangular lattice.

\section{SUMMARY AND DISCUSSION}

We have investigated finite-size scaling of an anisotropic two-dimensional ferromagnetic Ising model on an infinite strip of width $L$ with periodic, antiperiodic, and free bc in the direction perpendicular to the 
direction of infinite extent. The model is realized on a triangular lattice with general couplings and lattice constants, so that the anisotropy may be realized by varying the lattice constants $d_{i}$ and/or the couplings $J_{i}$. This allows for different microscopic realizations of identical anisotropic bulk long-distance correlations near the bulk critical point and therefore for a limited test of universality.

We find that the asymptotic scaling behavior of the finite-size contribution to the free energy per correlation volume may be described by a function of only one suitably defined scaling variable, cf. Eqs. (55) and (84). For periodic and antiperiodic bc this holds additionally for the surface and excess free energy densities per correlation volume, cf. Eqs. (56) and (57), while, for free bc, logarithmic violations of scaling prevent the scaling behavior of the surface contribution to the free energy density.

For periodic, antiperiodic, and free bc, we provide exact scaling functions $\mathcal{G}^{(\mathrm{p})}, \mathcal{G}^{(\mathrm{a})}$, and $\mathcal{G}^{(00)}$ of the finite-size contribution to the free energy density in Eqs. (59) and (86) and scaling functions $\mathcal{A}^{(\mathrm{p})}$ and $\mathcal{A}^{(\mathrm{a})}$ of the excess free energy density in Eq. (61). We find that these functions only depend on variables related to long-distance correlations. We have chosen these long-distance variables to be the scaling variable $\tilde{x}$ representing the ratio of the width $L$ of the strip and the bulk correlation length in the corresponding direction (for $T>T_{\mathrm{c}}$; for $T<T_{\mathrm{c}}$ its analytic continuation is employed) as well as the aspect ratio $r$ and the orientation angle $\theta$ of the bulk correlation lengths ellipse. Consequently, the critical Casimir amplitudes $\Delta^{(\mathrm{p})}, \Delta^{(\mathrm{a})}$, and $\Delta^{(00)}$ provided in Eqs. (65) and (87) depend only on $r$ and $\theta$. Since our results for $\mathcal{G}$, $\mathcal{A}$, and $\Delta$ are independent of the microscopic realization of the long-distance physics, we conjecture that they are universal functions of their respective arguments. That is, we expect identical functions to result for other members of the bulk two-dimensional Ising universality class for the same geometry and corresponding bc.

To understand the behaviors of the Ising systems described above, we have investigated general anisotropic systems on a $d$-dimensional film with the same bc as investigated for the two-dimensional Ising model. We assume that the free energies under consideration exhibit scaling. Due to the choice of geometry and bc, these systems have the simplifying property that their geometry and bc are invariant under a shear transformation relating them to a corresponding isotropic system. We find that the free energies per correlation volume of such systems depend on only one suitably chosen scaling variable, cf. Eqs. (114) and (124). The relations between the scaling functions of the isotropic and the anisotropic systems are provided in Eqs. (117) and (126) and reproduce for $d=2$ the relations found for the twodimensional Ising model. For ++ and $0+$ bc, where no results for the triangular lattice Ising model are available, we point out that these relations, together with the explicitly known isotropic results (130), lead to predictions for the corresponding anisotropic scaling functions of the finite-size contribution to the free energy density of the two-dimensional Ising model. We give explicit results for the corresponding critical Casimir amplitudes in (131).

We have only treated geometries and bc that are invariant under the shear transformation that relates the system under investigation to a system with isotropic bulk correlation lengths. In other cases we cannot expect to express scaling functions for the anisotropic case in terms of an isotropic-case scaling function with the same geometry and bc. Rather, the isotropic scaling functions will depend on the geometry and bc obtained by transforming the anisotropic to the isotropic case and the anisotropy will no longer be represented by mere geometric factors as in (59)-(61), (77), (86), (117), (123), and (126). However, we still expect such scaling functions to be universal functions of their arguments. Within a given bulk universality class, they should depend only on the scaling variable(s) used for the isotropic case, the asymptotic longdistance anisotropy represented by the matrix $\overline{\mathbf{A}}$ from (103), the geometry, and the bc. Hence we suggest that a quantity is universal, if it depends only on the bc and on macroscopic physical observables such as the geometry and the macroscopic near-critical correlation lengths, but not on the particular microscopic realization from which the anisotropic long-distance critical behavior originates. Such a quantity should therefore be identical among the members of the bulk universality class under consideration. For practical measurements of, e.g., scaling functions, it is no longer sufficient to measure one correlation length as in the isotropic case. Rather, the measurement of correlation lengths in a sufficient number of directions is necessary to allow for the determination of the matrix $\overline{\mathbf{A}}$ through (103).

Let us put our arguments in perspective with the interpretations of [16-19]. From our arguments follows that it is not necessary to define universality only after a shear transformation to an isotropic system, as Diehl and Chamati suggest [19]. As noted in Sec. III A, Chen and Dohm [16-18] define their matrix $\overline{\mathbf{A}}$ through microscopic parameters in the Hamiltonian so that, at least for lattice models, their matrix is only an approximation to our definition which relates $\overline{\mathbf{A}}$ through (103) to the asymptotic physical correlation lengths. Thus the dependence of scaling functions on their matrix $\overline{\mathbf{A}}$ will, in general, not be universal, since it depends on the microscopic realization of the anisotropy. In this sense it is correct when Chen and Dohm note that any dependence of physical quantities on their anisotropy matrix $\overline{\mathbf{A}}$ is nonuniversal [16-18]. In contrast, we suggest here that quantities that are universal for the isotropic case, merely acquire an additional universal dependence on $\overline{\mathbf{A}}$ from (103) for the anisotropic case. The parameters describing $\overline{\mathbf{A}}$ are called nonuniversal in [18]. This is correct in the sense that the relation of $\overline{\mathbf{A}}$ from [16-18] to our $\overline{\mathbf{A}}$ depends on the model under consideration. However, with our definition, $\overline{\mathbf{A}}$ is merely an argument of, e.g., a scaling function, and should be viewed on the same level as the scaling variable $\tilde{x}$. It is then only the scal- 
ing functions, whose universality can be tested and not that of their arguments $\tilde{x}$ and $\overline{\mathbf{A}}$. Similar arguments hold for the parameters describing the shear transformation, which are called nonuniversal in $[18,19]$. This transformation merely describes a relation between different physical situations (or different interpretations of the same statistical model), not necessarily one of them being isotropic. With our definitions, its classification as universal or nonuniversal is not a meaningful question.

Our last argument concerns the validity of two-scale factor universality. Since $\overline{\mathbf{A}}$ is a scale-free quantity, our universality interpretation does not interfere with twoscale factor universality in a formulation including the field $h$ conjugate to the order parameter. For definiteness, consider again film geometry with given bc and assume that the free energy density exhibits scaling. Then we expect the universal isotropic scaling function $[3,5,6]$

$$
L^{d} f_{s}(t, h, L)=\mathcal{F}\left(C_{1} t L^{1 / \nu}, C_{2} h L^{\beta \delta / \nu}\right),
$$

with nonuniversal scale factors $C_{1}$ and $C_{2}$ and critical exponents $\beta$ and $\delta$ to be generalized to an anisotropic universal scaling function

$$
L^{d} f_{s}(t, h, L)=\mathcal{F}\left(C_{1} t L^{1 / \nu}, C_{2} h L^{\beta \delta / \nu}, \overline{\mathbf{A}}, \hat{\mathbf{n}}\right),
$$

where the choice $C_{1}=\xi_{+, 0}^{(L)^{-1 / \nu}}$ makes the first argument identical to $\tilde{x}$ in (8) and leads to the $h=0$ limit $\mathcal{F}(\tilde{x}, 0, \overline{\mathbf{A}}, \hat{\mathbf{n}})=\mathcal{F}(\tilde{x}, \overline{\mathbf{A}}, \hat{\mathbf{n}})$, compare (110). Since no new nonuniversal scale factor needed to be introduced into Eq. (134) as compared to the isotropic case, two-scale factor universality remains valid. We have not quantitatively considered this case, since no exact results for the free energy of the two-dimensional Ising model with a nonzero magnetic field are available.

As an outlook, it would be interesting to investigate systems that do not exhibit the simplifying feature of both invariant geometry and bc under shear transformations. A possible example is the critical Binder cumulant for a two-dimensional ferromagnetic Ising model. Precision results for this quantity are available for a square lattice in a square geometry with periodic bc and a $45^{\circ}$ anisotropy that is caused by a ferromagnetic $[31,32]$ or antiferromagnetic [33] coupling on one of the lattice diagonals, i.e., by different microscopic realizations. For recent results of such an investigation, see Ref. [34].

\section{Acknowledgments}

The author is grateful to V. Dohm for suggesting this investigation and for helpful discussions and correspondence.

\section{Appendix A: Integrals and sums}

Here we collect some mathematical results needed for the determination of the scaling functions of the free en- ergy in Sec. II C 1.

On the one hand, we need, for sufficiently well-behaved functions $f$, the large- $n$ results

$$
\begin{aligned}
\int_{a}^{b} d x f(x)= & \sum_{j=1 / 2}^{n-1 / 2} f\left(x_{j}\right) \delta+\frac{1}{24}\left[f^{\prime}(b)-f^{\prime}(a)\right] \delta^{2}+\mathcal{O}\left(\delta^{3}\right), \\
\int_{a}^{b} d x f(x)= & \sum_{j=0}^{n-1} f\left(x_{j}\right) \delta+\frac{1}{2}[f(b)-f(a)] \delta \\
& -\frac{1}{12}\left[f^{\prime}(b)-f^{\prime}(a)\right] \delta^{2}+\mathcal{O}\left(\delta^{3}\right),
\end{aligned}
$$

respectively, where $\delta \equiv(b-a) / n$ and $x_{j} \equiv a+j \delta$.

On the other hand, using the method of residues, we obtain in the large- $n$ limit for real $x$

$$
\begin{aligned}
& \frac{1}{\pi} \int_{0}^{n \pi} d \omega \sqrt{x^{2}+4 \omega^{2}}-\sum_{k=0}^{n-1} \sqrt{x^{2}+[(2 k+1) \pi]^{2}} \\
& =\frac{\pi}{12}+I^{(+)}(x), \\
& \frac{1}{\pi} \int_{0}^{\left(n-\frac{1}{2}\right) \pi} d \omega \sqrt{x^{2}+4 \omega^{2}}-\sum_{k=1}^{n-1} \sqrt{x^{2}+(2 k \pi)^{2}} \\
& =\frac{|x|}{2}-\frac{\pi}{6}+I^{(-)}(x),
\end{aligned}
$$

with $I^{( \pm)}$defined in (50).

The results (A1) and (A2) allow us to obtain Eq. (49) from Eq. (48).

\section{Appendix B: Isotropic scaling functions}

Here we relate the scaling functions $\mathcal{G}$ and $\mathcal{A}$ to published scaling functions of the Casimir force for the isotropic two-dimensional Ising model in infinite-strip geometry. This allows us to verify the isotropic limits of our results for $\mathcal{G}^{(\mathrm{p})}, \mathcal{G}^{(\mathrm{a})}$, and $\mathcal{G}^{(00)}$ and to derive the scaling functions $\mathcal{G}_{\text {iso }}^{(00)}$ and $\mathcal{G}_{\text {iso }}^{(0+)}$ that can subsequently be used for predictions about the anisotropic case as explained in Sec. III B 2.

For the isotropic case, the Casimir force of a $d$ dimensional film system of thickness $L$ is defined by

$$
\begin{aligned}
F_{\text {Cas }, \text { iso }}(T, L) & \equiv-\frac{\partial\left[L f_{\text {iso,ex }}(T, L)\right]}{\partial L} \\
& =-\frac{\partial\left[L f_{\text {iso }, \mathrm{fs}}(T, L)\right]}{\partial L}
\end{aligned}
$$

where the second equality reflects the fact that surface contributions do not contribute to the Casimir force. If its singular part $F_{\text {Cas,iso,s }}$ exhibits scaling, a corresponding scaling function $X_{\text {iso }}$ may be defined in the asymptotic critical domain, so that

$$
\begin{aligned}
& L^{d} F_{\text {Cas }, \text { iso }, \mathrm{s}}(t, L)=X_{\text {iso }}(\tilde{x}) \\
& =(d-1) \mathcal{G}_{\text {iso }}(\tilde{x})-\nu^{-1} \tilde{x} \frac{d \mathcal{G}_{\text {iso }}(\tilde{x})}{d \tilde{x}},
\end{aligned}
$$


where (6b) and (8) have been used. With (21), this may be expressed, for the two-dimensional Ising model, as

$$
L^{2} F_{\text {Cas, }, \text { iso }, \mathrm{s}}(t, L)=X_{\text {iso }}(\tilde{x})=-\tilde{x}^{2} \frac{d\left[\mathcal{G}_{\text {iso }}(\tilde{x}) / \tilde{x}\right]}{d \tilde{x}} .
$$

\section{Periodic and antiperiodic bc}

Here we use results from the literature for $X_{\text {iso }}^{(\mathrm{p} / \mathrm{a})}$ to verify the isotropic limits (62) of the scaling functions $\mathcal{G}^{(\mathrm{p} / \mathrm{a})}$ provided in (59). Rescaling $\omega \rightarrow|x| \omega$ in (50), applying (B3) to the resulting expression for $\mathcal{G}_{\text {iso }}^{(\mathrm{p} / \mathrm{a})}(\tilde{x})$ and subsequently scaling back according to $\omega \rightarrow \omega /|x|$ gives

$$
\begin{aligned}
X_{\text {iso }}^{(\mathrm{p} / \mathrm{a})}(\tilde{x})= & \frac{1}{2 \pi} \int_{0}^{\infty} d \omega \sqrt{\tilde{x}^{2}+\omega^{2}} \\
& \times\left\{\begin{array}{l}
{\left[\tanh \left(\sqrt{\tilde{x}^{2}+\omega^{2}} / 2\right)-1\right]} \\
{\left[\operatorname{coth}\left(\sqrt{\tilde{x}^{2}+\omega^{2}} / 2\right)-1\right]}
\end{array}\right.
\end{aligned}
$$

where the upper and lower expressions hold for periodic and antiperiodic bc, respectively. Corresponding critical values are

$$
X_{\text {iso }}^{(\mathrm{p} / \mathrm{a})}(0)=\left\{\begin{array}{l}
-\pi / 12, \\
\pi / 6 .
\end{array}\right.
$$

The upper result in (B4) agrees numerically with the Ising curve plotted in Fig. 15 of Ref. [35] ( $x$ there is identical to our $\tilde{x}$ ). The upper and lower results in (B4) agree with Eqs. (3.16) and (3.19), respectively, of Ref. [36] (x there is identical with our $\tilde{x} / 2$ ). The upper result in (B5) agrees with Eq. (1) in Ref. [37], with Eq. (3) in Ref. [38], and with Eq. (58) in Ref. [27].

\section{Fixed and free bc}

Here we use results from Refs. [39] and [40] for the Casimir force scaling functions $X_{\text {iso }}^{(00)}, X_{\text {iso }}^{(++)}$, and $X_{\text {iso }}^{(0+)}$, for the isotropic two-dimensional Ising model in infinitestrip geometry to derive the scaling functions $\mathcal{G}_{\text {iso }}^{(00)}$, $\mathcal{G}_{\text {iso }}^{(++)}$, and $\mathcal{G}_{\text {iso }}^{(0+)}$. From Eqs. (2.9) and (2.13) in Ref. [39] follows for the isotropic-case Casimir force scaling functions for ++ and $00 \mathrm{bc}$ in our notation ( $X$ there is identical to our $\tilde{x} / 2$ )

$$
\begin{aligned}
X_{\text {iso }}^{(++)}(\tilde{x}) & =X_{\text {iso }}^{(00)}(-\tilde{x}) \\
& =-\frac{1}{\pi} \int_{0}^{\infty} \frac{d \omega \sqrt{\tilde{x}^{2}+\omega^{2}}}{1+\frac{\sqrt{\tilde{x}^{2}+\omega^{2}}-\tilde{x}}{\sqrt{\tilde{x}^{2}+\omega^{2}}+\tilde{x}} e^{2 \sqrt{\tilde{x}^{2}+\omega^{2}}}} .
\end{aligned}
$$

Equivalent results are obtained from Eq. (3) in Ref. [40] for both 00 and ++ bc $(x$ there is identical to our $\tilde{x})$ [41] and from Eq. (3.26) in Ref. [36] for $00 \mathrm{bc}(x$ there is identical to our $\tilde{x} / 2$ ), while the results from Sec. 12.1.2 of [9] for $X_{\mathrm{Cas}}^{(o, o)}(x)$ and $X_{\mathrm{Cas}}^{(+,+)}(x)(x$ there is our $2 \tilde{x})$ are missing a factor $1 / 4$. Using (B3) and observing that, for large positive or negative $\tilde{x}$, the scaling function $\mathcal{G}$ contains by definition no surface or interface terms, i.e., no terms linear in $\tilde{x}$, we obtain from (B6) by elementary integration the scaling functions $\mathcal{G}_{\text {iso }}^{(00)}$ provided in (88) and (130a) and $\mathcal{G}_{\text {iso }}^{(++)}$provided in (130a) .

From Eq. (3) in Ref. [40] ( $x$ there is identical to our $\tilde{x})$, we obtain [41]

$$
\begin{aligned}
X_{\text {iso }}^{(0+)}(\tilde{x}) & =\frac{1}{2 \pi} \int_{0}^{\infty} d \omega \sqrt{\tilde{x}^{2}+\omega^{2}}\left(\operatorname{coth} \sqrt{\tilde{x}^{2}+\omega^{2}}-1\right) \\
& =X_{\text {iso }}^{(\mathrm{a})}(2 \tilde{x}) / 4,
\end{aligned}
$$

with $X_{\text {iso }}^{(\text {a) }}$ from (B4). With (B3) and taking again into account the absence of large- $|\tilde{x}|$ linear terms in $\mathcal{G}$, elementary integration leads immediately to $\mathcal{G}^{(0+)}$ as provided in (130b).
[1] M.E. Fisher, Rev. Mod. Phys. 46, 597 (1974).

[2] M.E. Fisher, in Critical Phenomena, Proceedings of the 1970 International School of Physics "Enrico Fermi," Course 51, edited by M.S. Green (Academic, New York, 1971), p. 1.

[3] V. Privman and M.E. Fisher, Phys. Rev. B 30, 322 (1984).

[4] V. Privman, Phys. Rev. B 38, 9261 (1988).

[5] V. Privman, in Finite Size Scaling and Numerical Simulation of Statistical Systems, edited by V. Privman (World Scientific, Singapore, 1990), p. 1.

[6] V. Privman, A. Aharony, and P.C. Hohenberg, in Phase Transitions and Critical Phenomena, edited by C. Domb and J.L. Lebowitz (Academic, New York, 1991), Vol. 14, p. 1.

[7] F. Wegner, Phys. Rev. B 5, 4529 (1972).

[8] M. Krech, The Casimir Effect in Critical Systems (World Scientific, Singapore, 1994).

[9] J.G. Brankov, D.M. Danchev, and N.S. Tonchev, Theory of Critical Phenomena in Finite-Size Systems-Scaling and Quantum Effects (World Scientific, Singapore, 2000).

[10] A. Gambasssi, J. Phys. Conf. Ser. 161, 012037 (2009).

[11] R.B. Griffiths, Phys. Rev. Lett. 24, 1479 (1970).

[12] A.D. Bruce, J. Phys. C 7, 2089 (1974).

[13] T.T. Wu, B.M. McCoy, C.A. Tracy, and E. Barouch, Phys. Rev. B 13, 316 (1976). 
[14] M. Campostrini, A. Pelissetto, P. Rossi, and E. Vicari, Phys. Rev. E 57, 184 (1998).

[15] H.W.J. Blöte, A. Compagner, and A. Hoogland, Physica A 141, 375 (1987).

[16] X.S. Chen and V. Dohm, Phys. Rev. E 70, 056136 (2004); 71, 059901(E) (2005).

[17] V. Dohm, J. Phys. A: Math. Gen. 39, L259 (2006).

[18] V. Dohm, Phys. Rev. E 77, 061128 (2008); 79, 049902(E) (2009); 82, 029902(E) (2010).

[19] See Sec. VI of H.W. Diehl and H. Chamati, Phys. Rev. B 79, 104301 (2009).

[20] See N.S. Tonchev, Phys. Rev. E 75, 031110 (2007) and references therein.

[21] X.S. Chen and V. Dohm, Phys. Rev. E 66, 016102 (2002); 66, 059901(E) (2002).

[22] X.S. Chen and V. Dohm, Eur. Phys. J. B 10, 687 (1999).

[23] J.O. Indekeu, M.P. Nightingale, and W.V. Wang, Phys. Rev. B 34, 330 (1986).

[24] The minus sign is missing in Eq. (A22) of Ref. [23].

[25] T.M. Liaw, M.C. Huang, S.C. Lin, and M.C. Wu, Phys. Rev. B 60, 12994 (1999).

[26] The minus sign in Eq. (66) of Ref. [25] needs to be replaced by a plus sign.

[27] A. Hucht, D. Grüneberg, and F.M. Schmidt, Phys. Rev. E 83, 051101 (2011).

[28] The "ln" is missing in the second term on the right hand side of Eq. (A15) of Ref. [23]. No expression for $\bar{a}$ was provided in Ref. [23]. We have simplified the expressions for $p_{ \pm}$.

[29] J.L. Cardy, in Phase Transitions and Critical Phenomena, edited by C. Domb and J. L. Lebowitz (Academic, New York, 1987), Vol. 11, p. 55.

[30] B. Kastening and V. Dohm, Phys. Rev. E 81, 061106 (2010).

[31] G. Kamienarz and H.W. Blöte J. Phys. A: Math. Gen. 26, 201 (1993).

[32] W. Selke and L.N. Shchur, J. Phys. A: Math. Gen. 38, L739 (2005).

[33] W. Selke and L.N. Shchur, Phys. Rev. E 80, 042104 (2009).

[34] B. Kastening, arXiv:1209.0105v1.

[35] O. Vasilyev, A. Gambassi, A. Maciołek, and S. Dietrich, Phys. Rev. E 79, 041142 (2009).

[36] J. Rudnick, R. Zandi, A. Shackell, and D. Abraham, Phys. Rev. E 82, 041118 (2010).

[37] H.W.J. Blöte, J.L. Cardy, and M.P. Nightingale, Phys. Rev. Lett. 56, 742 (1986).

[38] I. Affleck, Phys. Rev. Lett. 56, 746 (1986).

[39] R. Evans and J. Stecki, Phys. Rev. B 49, 8842 (1994).

[40] D.B. Abraham and A. Maciołek, Phys. Rev. Lett. 105, 055701 (2010).

[41] The relation published after Eq. (3) in Ref. [40] for the quantity $X^{ \pm}(y)$ has an incorrect distribution of signs and should correctly read $X^{ \pm}(y)=\lambda(x, u) \pm\left(x-2 e^{2 K_{c}} y\right)$. The author is grateful to A. Maciołek for helping to clarify this issue. 